\title{
Modelling of irregular-shaped particles and microstructural development of
}

\section{Portland cement}

${ }^{\mathrm{c}}$ State Key Laboratory of Geomechanics and Deep Underground Engineering, China University of Geomatic Engineering, University College London, London WC1E 6BT, UK

${ }^{a}$ School of Materials Science and Engineering, Southeast University, Nanjing 211189, China

${ }^{\mathrm{b}}$ Advanced and Innovative Materials (AIM) Group, Department of Civil, Environmental and

Abstract: The shape of cement powder particles plays a crucial role in particle packing and hydration process of cement. However, cement powder is generally regarded as sphere for simplification in most cement hydration models. This paper aims to investigate the influence of cement particle shapes on hydration of Portland cement and microstructure of cement paste. A novel central growth model along with a particle packing algorithm is developed to generate cement particles with irregular shapes and reconstruct initial 3D microstructures of Portland cement. Afterwards, the hydration process of cement with different shapes are simulated using CEMHYD3D model. The results indicate that the generated irregular-shaped particles precisely reproduce the real cement particles in terms of surface area, particle size distribution and geometry. The effect of particle shapes on hydration is significant due to the difference in surface area and geometric discrepancy, whereas this effect becomes less obvious with decreasing water-to-cement ratio.

Keywords: Cement hydration; Particle shape; Particle packing; Microstructure; Setting; Connectivity

\footnotetext{
* Corresponding authors. E-mail address: zhangys279@163.com (Y. Zhang)
} 


\section{Introduction}

Many natural and man-made materials composed of particles typically have random irregular shapes rather than an inherent shape, which leads to an increasing number of interests in the investigation of the influence of particle shapes on microscopic and macroscopic properties of granular media [1]. With regard to cement-based materials, the shapes of cement powder particles and aggregates have been shown to play a critical role in their workability, mechanical performance and durability [2-5]. Cement powders sharing similar clinker, gypsum and fineness have different water demands, setting behaviour and viscosity as different grinding techniques are used [2]. These discrepancies are attributed to the different particle shapes of cement. Therefore, an accurate characterisation and reconstruction of real irregular particle shapes is a crucial aspect in better understanding of hydration process, microstructural evolution and mechanical properties development of cement-based materials.

In recent years, different advanced techniques including $3 \mathrm{D}$ laser ranging $[6,7]$ and $\mathrm{X}$-ray computed tomography (XCT) [8] have been used to characterise and analyse the 3D shapes of coarse aggregates in concrete. Unlike coarse aggregates, the size of cement powder particles ranges from a few tenths of a micrometer to dozens of micrometers. It is still quite challenging to accurately capture the individual cement particle shapes in 3D using these techniques due to their limited resolution as well as the complicated and prohibitive sample preparation process. For example, there should be at least 10 voxels in any one dimension of a particle in order to obtain this particle's shape that can only be achieved using the best XCT synchrotron sources [9]. Computer simulation may provide a suitable methodology to reconstruct and analyse the 3D shapes of cement powder particles without above-mentioned drawbacks. Meanwhile, the only way to overcome the conundrum of investigating the influence of specific cement particle shapes on hydration process 
and microstructural evolution of cement pastes is numerical simulation to date.

Over the past few decades, several cement hydration models validated by experiments have been proposed, such as HYMOSTRUC3D [10], $\mu$ ic [11], DuCOM [12] and CEMHYD3D [13, 14], which give the possibility of investigating the relationship between cement particle shapes and cement hydration process in a quantitative manner. These models can not only capture the cement hydration process including the evolution of each phase (e.g., hydration products, anhydrous cement and capillary pore) but also the long-term properties including mechanical strength and transport properties. Nevertheless, in almost all these hydration models, the cement particles are simply assumed spherical in shape, which is far from the real situation that cement particle shapes are irregular in general. As such, the accuracy of simulation results using these models would be significantly affected. Therefore, it is essential to take irregular particle shapes into consideration in order to provide more accurate simulation of hydration and microstructure of cement-based materials. Although an increasing number of studies have been undertaken to explore the effect of irregular shapes of aggregate on properties of cement-based materials, unfortunately, to the best of authors' knowledge, there are only very few studies related to the influence of cement particle shapes on cement hydration. Researchers from National Institute of Standard and Technology (NIST) are the pioneers to capture the 3D shapes of cement particles of a standard reference cement, CCRL-133, using XCT techniques and reconstruct 3D cement particles with irregular shapes by spherical harmonic function for replacing the spherical cement powders in CEMHYD3D [2, 9, 15]. The spherical harmonic function method could successfully reproduce the real cement particles, while it is only suitable for medium and large cement particles due to the limitation of resolution of XCT images, i.e., $0.95 \mu \mathrm{m} /$ voxel. Moreover, the reconstructed particle library is only available for a specific reference cement due to the complex, time-consuming and often very expensive 
experiments. Thus, further studies are needed to reconstruct and analyse real irregular shapes of cement particles and complement our knowledge in the field.

The main purpose of this paper is to reconstruct the irregular-shaped particles and investigate the influence of shapes of cement particles on Portland cement hydration process. A novel central growth model (CGM) based on the discrete method is proposed to rebuild cement particles with random irregular shapes. The theory of CGM and relevant procedures of particle packing for generating 3D representative volume element (RVE) of irregular-shaped cement particles are described in detail. The obtained initial microstructure of cement is subsequently incorporated into the CEMHYD3D model to simulate the hydration and setting of irregular-shaped cement. A series of experiments are carried out to acquire the input parameters for simulations and measure some properties associated with hydration of a Chinese Portland cement (similar to ASTM Type I Portland cement) for validation of simulations including hydration heat, degree of hydration and setting behaviour. Based on the input parameters obtained from experiments and the reconstructed RVE accounting for irregular-shaped particles, parametric analysis and sensitivity analysis are undertaken to estimate the effect of particle shape on particle packing such as surface area, particle size distribution (PSD) and geometry, and cement hydration process including hydration heat, degree of hydration and connectivity of solid phases.

\section{Initial microstructure model of irregular-shaped cement}

\subsection{General}

In order to introduce irregular-shaped cement particles into cement hydration model, it is necessary to produce 3D irregular shapes of cement powder particles and then carry out random packing of these particles in a RVE. Herein, a central growth model based on the discrete method and cellular automata theory is proposed and developed to achieve these purposes. 
The discrete-based method is an important approach for reconstructing objects in imaging

techniques. 2D and 3D images are pixel and voxel based respectively. No matter how complex the single object is in shape, its shape can be represented using discrete basic elements. In turn, the quality of pixels or voxels can affect the similarity of the reconstructed shape and the shape of real object. Although the discrete-based method has a noticeable resolution-dependent limitation, as compared to the vector-based method for generating irregular-shaped particles, it can easily determine the geometric attributes and obtain an effective random packing process. For example, it is difficult to estimate the surface area of a vector-based random-shaped particle using traditional algorithms [16]. Regarding the packing algorithm of irregular-shaped particles, the mathematical decision procedure of overlapping between particles using vector-based method is too complicated and time-consuming $[17,18]$. Furthermore, the discrete-based cement hydration model has been successfully employed to simulate the hydration process and microstructural development of cement-based materials [14]. In this work, the procedure used to simulate cement hydration process follows the similar rules that have been applied to the existing discrete-based hydration model, e.g., CEMHYD3D, in which the RVE is digitized into cubic voxels representing cement particles and initial packing state composed of these voxels. Thus, the discrete-based method for producing cement particles with irregular shapes can directly meet the rules of the cement hydration model without digitizing the reconstructed particles again, if the spatial resolution of particles is identical to that of RVE. In contrast, the obtained virtual microstructure of cement-based materials from the vector-based cement hydration models, e.g., HYMOSTRUC3D and $\mu \mathrm{ic}$, needs to be digitized or meshed prior to its incorporation into other numerical models for determining physical and mechanical properties of cement-based materials $[11,19,20]$. Therefore, the discrete-based method is chosen and used to produce irregular-shaped cement particles in this work because of its 
attractive advantages.

Cellular automation is a discrete method that has been widely used in microstructure modelling, mathematics, physics, complexity science and other areas [21]. Cellular automation consists of three basic elements, i.e., cells, evolution rules and relationship between adjacent cells. Cellular automata algorithm has been successfully implemented in the CEMHYD3D cement hydration model $[13,14]$. It was found that the voxel size and different relationship between neighbouring voxels have strong influences on the simulated pore structure, solid percolation and hydration rate of cement paste using CEMHYD3D [22]. As shown in Fig. 1, there exists three relationships between neighbouring voxels of the target voxel in 3D that are referred to as face-to-face, edge-to-edge and point-to-point neighbour models, respectively. In face-to-face neighbour model, the voxels are identified as neighbours of the target voxel if they have sharing faces with the target voxel. Similarly, the neighbours are defined as the voxels with sharing faces and edges in edge-to-edge neighbour model, and sharing faces, edges and points in point-to-point neighbour model, respectively. For face-to-face, edge-to-edge and point-to-point neighbour models, the number of neighbours is 6,18 and 26 , respectively.

Fig. 2 shows a detailed flow chart of the generation of microstructure of irregular-shaped cement using the proposed discrete-based CGM (to be described in detail in the following sections), the entire procedure of which can be divided into five steps. Steps I and II are related to the generation of individual irregular-shaped cement particles, while Steps III to V are associated with the random packing of these individual particles. For the sake of simplicity, in some cases 2D images are used to better explain the algorithm and implementation in detail. Prior to the reconstruction of cement particles, some basic information in relation to hydration system, e.g., the RVE size, spatial resolution, PSD and w/c ratios are taken into consideration and incorporated into 
the model. Afterwards, the corresponding characteristics of particles to each diameter interval including the particle number can be summarised and analysed. The diameter of irregular-shaped particles in CGM is equivalent to that of spherical particles based on the volume of particles regardless of specific shapes.

\subsection{Generation of single irregular-shaped particle}

Herein, how to produce the individual particles with irregular shapes using CGM is introduced in detail. As seen in Fig. 2, the entire procedure of generating single irregular-shaped particles consists of following two steps.

Step I: Initially, a 3D discrete box with the same resolution as that of RVE is constructed and used as growing space, which should be large enough to accommodate single particle. In general, we choose $2 / 3$ time size of RVE as a typical growing space when modelling multi-size cement particles. Subsequently, the coordinates of the central point in this discrete growing space and a set of default eigenvectors (or eigenvector library) for growing irregular-shaped particles are transferred into a list. It should be pointed out that the edge-to-edge neighbour model is adopted, which means the target cell has 8 neighbouring pixels in $2 \mathrm{D}$ and 18 neighbouring voxels in $3 \mathrm{D}$, as shown in Fig. 2. The values (0-100) of the eigenvector in each square (or cube) represent the growing probabilities of the particle in the corresponding dimension. For example, considering the central blue pixel as the original point, the value of 29 at the point of $(1,1)$ implies that all pixels have a probability of 0.29 to become a part of this particle (blue) in this dimension.

Step II: To better understand the generation process of an irregular-shaped particle, a simple example of $2 \mathrm{D}$ growing method in $15 \times 15$ pixels discrete space is shown in Fig. 2. Firstly, the coordinates of the first node in the list (i.e., central point) are read and the corresponding pixel is turned into a blue particle pixel, as shown in Fig. 2a. Its eight neighbouring pixels highlighted in 
yellow colour are then activated and a set of eight probability values between 0 and 100 are generated for them sequentially based on a Monte Carlo simulation. The eight probability values are then compared with the corresponding characteristic values of the special irregular particles, respectively. If the pointed cell has a probability value smaller than its corresponding characteristic one, the cell would be turned into a part of the particle. As seen in Fig. 2b, five neighbours among the eight neighbouring pixels are turned into cement particle and their coordinates are delivered into the list one by one. Repeating the steps shown in Figs. $2 \mathrm{a}$ and $2 \mathrm{~b}$ for these five neighbouring pixels, more neighbouring pixels can be classified into the cluster of cement particle, as shown in Figs. 2c and $2 \mathrm{~d}$. These steps are continuously operated on the activated neighbouring pixels until the target area of this particle is achieved. Fig. 2e illustrates an obtained irregular cement particle with 67 pixels. The generation of particles with irregular shapes in $3 \mathrm{D}$ complies with the similar algorithm and implementation process that just needs to change the number of neighbours. Fig. $2 \mathrm{f}$ shows an individual particle with irregular shape in $3 \mathrm{D}$, where the green voxels represent the simulated cement particle and the remaining voxels denote empty space.

Although the magnitude of numbers in a certain eigenvector is associated with the growth probability in the corresponding dimension for each voxel in CGM, there seems to be no relevant quantitative relationship between different irregular particle shapes as found by a large number of attempts. However, the same eigenvector may produce self-similar particles. Fig. 3 shows the irregular-shaped particles reconstructed through different attempts using same eigenvector and various constituent voxels. The phenomenon of self-similarity can be observed from the simulated particles with similar orientation that is independent on the number of consitituent voxels, while the local shape discrepency exists between each analogical particles. This implies that it is possible to generate similar particles with local shape discrepency using same eigenvector. As a consequence, 
such similarity of irregular-shaped particles generated with same eigenvector enables us to develop an eigenvector library of 3D shapes once the particle shape descriptors are determined.

Over the past few decades, a number of efforts have been made to characterise and describe the particle shapes and a few particle descriptor parameters for 3D shape have been proposed for the purpose of 3D shape classification [23-26]. Among them, sphericity is one of the most widely used particle shape parameters that is defined as [27]:

$$
S_{A}=\frac{A_{s}}{A}
$$

where $A$ is the particle's surface area and $A_{s}$ is the surface area of a sphere that has same volume.

From Eq. (1) and the isoperimetric inequality, it can be easily derived that $0<S_{A} \leq 1$, the upper bound of which can only be attained for sphere. For discrete irregular particles, the voxel-by-voxel scanning technique can be used to accurately calculate volume and surface area [28]. Fig. 5 shows the relationship between sphricity and number of constituent voxels for particles with five representative shapes that are illustrated in Fig. 4. It can be seen clearly that the sphericity of the reconstructed particles dramatically fluctuates as the number of constituent voxels is less than 1000 in general, but gradually reaches fixed values when the number of voxels exceeds this threshold. In detail: the sphericity values of M1 and M4 are higher than those of M2 and M3, which is consistent with the results presented in Fig. 4. The quantitative analysis demonstrates the feasiblitiy of creating a full range of virtual irregular particle shape library. However, the sphericity seems not accurate enough to serve as the sole criterion due to some errors. For example, the geometry of particle M4 looks much more like a sphere than that of M1, while their values of sphericity are opposite. In addition, the digitized sphere (DS) composed of a large number of voxels has an average sphericity of 0.67 that is far less than 1 . This can be attributed to that the roughness and planar surfaces of particles consisting of cubic voxels increase with increasing number of 
voxels, and accordingly the surface area is increased [29]. Therefore, besides sphericity, another shape descriptor is required to accurately classify the 3D shape of irregular particles.

Another widely used particle shape descriptor is dimensions of irregular particles, such as length $L$, thickness $T$ and width $W$ along the particle's longest axis, shortest axis and axis perpendicular to both $L$ and $T$ [24, 30, 31]. However, such definition of triaxial dimensions exists inherent drawbacks. One possible drawback is that small protuberances on the irregular particles can significantly enlarge the values of $L, W$ and $T$ [26]. Such unstable phenomenon is much more significant among discrete particles generated by CGM. It can be found from a series of attempts to $L, W$ and $T$ that there is a dramatic difference among the particles produced using same eigenvector and each attempt may lead to a local discrepancy in the similarly-shaped particles. In order to overcome this disadvantage, other sets of three orthagonal dimensions can be employed by using moments of inertia.

For a rigid body, the principal axes of inertia are a set of orthogonal axes passing through the centre of mass of the body. These axes are related to the object's mass distribution and rotation in such a way that when the body rotates free of torques about any of these axes, the angular momentum is not transferred to any other axis. If the rigid body is homogeneous, the values of these three principal moments have a direct relation with its shape. In detail: if the three values are almost identical, this particle is highly symmetrical, like a sphere or cube. If two of the three principal moments values are close to each other and larger than the third one, the particle shape is "plate like". If two values are close to each other but smaller than the third one, the shape is "needle like" [26]. In order to acquire a volume-independent shape descriptor, the orthogonal axial lengths of the moment of inertia of an equivalent inertia ellipsoid are used as references. Hereinafter, three semi-axis dimensions of equivalent ellipsoid are referred to as the maximum length $a$, intermediate 
length $b$, and minimum length $c(a \geq b \geq c)$. The combination of normalized ratios of $a / c$ and $b / c$ is another sorting criterion. The procedure to derive $a, b$ and $c$ is described in detail below.

Three principal axes are in the direction of the eigenvectors of inertia tensor that is a symmetric and second rank tensor. For a discrete particle produced by CGM, the second tensor satisfies the following equation:

$$
I=\left(\begin{array}{ccc}
I_{x x} & I_{x y} & I_{x z} \\
I_{y x} & I_{y y} & I_{y z} \\
I_{z x} & I_{z y} & I_{z z}
\end{array}\right)
$$

where $I_{x x}, I_{y y}$ and $I_{z z}$ represent the second moments, and $I_{x y}, I_{x z}, I_{y x}, I_{y z}, I_{z x}$ and $I_{z y}$ denote the product moments $\left(I_{x y}=I_{y x}, I_{x z}=I_{z x}\right.$ and $\left.I_{y z}=I_{z y}\right)$, respectively. The second moments and product moments can be expressed as:

$$
\begin{aligned}
& I_{x x}=\sum m_{i}\left(y_{i}^{2}+z_{i}^{2}\right) ; I_{y y}=\sum m_{i}\left(z_{i}^{2}+x_{i}^{2}\right) ; I_{z z}=\sum m_{i}\left(x_{i}^{2}+y_{i}^{2}\right) \\
& I_{x y}=-\sum m_{i}\left(x_{i} y_{i}\right) ; I_{x z}=-\sum m_{i}\left(x_{i} z_{i}\right) ; I_{y z}=-\sum m_{i}\left(y_{i} z_{i}\right) \\
& m_{i}=\rho_{i} V_{i}
\end{aligned}
$$

where $\left(x_{i}, y_{i}, z_{i}\right)$ is the coordinate of voxel $i$ belonging to the irregular-shaped particle, $m_{i}, \rho_{i}$ and $V_{i}$ denote the mass, density and volume of voxel $i$, respectively.

The three principal moments of the irregular-shaped particle can be determined by solving the following equation:

$$
\left|\begin{array}{ccc}
I_{x x}-\lambda & I_{x y} & I_{x z} \\
I_{y x} & I_{y y}-\lambda & I_{y z} \\
I_{z x} & I_{z y} & I_{z z}-\lambda
\end{array}\right|=0
$$

From Eq. (6), the three roots for $\lambda\left(\lambda_{\max } \geq \lambda_{\text {int }} \geq \lambda_{\min }\right)$ can be obtained and these values are identical to three principal moments respectively. Accordingly, the equations of three principal moments of ellipsoid can be found in [32] as follows:

$$
I_{a}=\left(b^{2}+c^{2}\right) / 5 ; \quad I_{b}=\left(a^{2}+c^{2}\right) / 5 ; \quad I_{c}=\left(a^{2}+b^{2}\right) / 5
$$


where $I_{a}, I_{b}$ and $I_{c}$ denote the principal moments along the axis of ellipsoid with half length of $a, b$ and $c$, respectively.

To extract the values of three semi-axis dimensions of equivalent ellipsoid with same principal moments as those of the irregular-shaped particle, Eqs. (6) and (7) can be combined to obtain the following equation:

$$
a=\sqrt{\frac{5}{2}\left(-\lambda_{\min }+\lambda_{\mathrm{int}}+\lambda_{\max }\right)} ; \quad b=\sqrt{\frac{5}{2}\left(\lambda_{\min }-\lambda_{\mathrm{int}}+\lambda_{\max }\right)} ; \quad c=\sqrt{\frac{5}{2}\left(\lambda_{\min }+\lambda_{\mathrm{int}}-\lambda_{\max }\right)}
$$

It should be mentioned that the reconstructed irregular-shaped cement particles are assumed to be homogeneous in this Section. The heterogeneity of them with different densities and multi-phases, including $3 \mathrm{CaO} \cdot \mathrm{SiO}_{2}\left(\mathrm{C}_{3} \mathrm{~S}\right), \quad 2 \mathrm{CaO} \cdot \mathrm{SiO}_{2}\left(\mathrm{C}_{2} \mathrm{~S}\right), \quad 3 \mathrm{CaO} \cdot \mathrm{Al}_{2} \mathrm{O}_{3} \quad\left(\mathrm{C}_{3} \mathrm{~A}\right)$ and $4 \mathrm{CaO} \cdot \mathrm{Al}_{2} \mathrm{O}_{3} \cdot \mathrm{Fe}_{3} \mathrm{O}_{4}\left(\mathrm{C}_{4} \mathrm{AF}\right)$, will be discussed in detail in Section 3.1 .

Fig. 6 shows the relationship between the normalized semi-axes lengths of the equivalent inertia ellipsoid of irregular-shaped particles and number of constituent voxels. It can be seen that both $a / b$ and $a / c$ ratios change with number of constituent voxels before the number of voxels reaches a certain threshold value. This tendency is similar to the relationship between particle sphericity and number of constituent voxels in Fig. 5. However, for the same type of particles, the threshold values of constituent voxels for $a / b$ and $a / c$ ratios are not identical to each other and also different from that for sphericity. In Fig. 6a, the threshold values of the most spherical M4 in $a$-axis direction is around 100, while that of M3 with the largest discrepany of length along $a$-axis is over 10000 . With respect to $b$-axis, Fig. $6 \mathrm{~b}$ also demonstrates a similar tendency, indicating that M4 with the least difference of length has loweast threshold value of about 100. On the contrary, M2 with the largest difference along $b$-axis shows the largest threshold value of over 5000. This means that the threshold value of constituent voxels in the corresponding direction increases with the length discrepancy. This is because when particle is less equiaxed, more constituent voxels are required to 
describe its features with large dimensional discrepancy [9]. In spite of existing fluctuating threshold values for particles with different shapes, the ratios of $a / c$ and $b / c$ both approach fixed values if there are sufficient constituent voxels. It obviously indicates that the particle generated using the dependent eigenvector has its inherent shape attributes that can be described by the normalized semi-axes dimensions of equivalent inertia ellipsoid.

With regard to the relationship between three semi-axes lengths and specific shapes in Figs. 4 and 6, the particle M1 has a mean ratio of $a: b: c$ of 1.43:1.31:1.00 and looks a lttle flat, while M3 with a mean ratio of $a: b: c$ of $3.21: 1.27: 1.00$ exhibits an elongated shape. M4 with a ratio of $a: b: c$ of 1.11:1.08:1.00 is much more spherical than M1, which means that the $a: b: c$ ratio can be used to effectively distinguish particles with simialr sphericity, e.g., M1 and M4. The intermediate particle is M2 that has a ratio of $a: b: c$ of 2.01:1.53:1. Although this equivalent inertia ellipsoid method can well classify irreugular particles based on their symmetry, it cannot distinguish the high symmetrical particles [25]. Therefore, the criteria for irregular-shaped particles should include two shape descriptors, i.e., sphericity and normalized semi-axes lengths of equivalent inertia ellipsoid, to overcome their respective deficiencies and create the shape library of particles.

\subsection{Irregular-shaped particle packing}

Once the particle shape library is built up, the random particles packing can be subsequently undertaken to reconstruct the initial 3D RVE of cement mixes with a desired water-to-cement (w/c) ratio. The random particle packing procedure includes three steps (see Fig. 2) that are described in detail as follows:

Step III: Due to the similar orientation of particles produced by the same eigenvector, the grown cement particle needs to be rotated by a random angle $\alpha$ in the growing space to keep its random orientation in the RVE. The rotated process is given as follows. Firstly, all particle voxels 
are revolved around the growing center point by an random angle $\beta([0,2 \pi])$ in $X Y$ plane. The same method is then used to rotate another random angle $\gamma([0,2 \pi])$ in $Y Z$ plane. As such, the coordinate of each voxel of cement particle $\left(x_{A}, y_{A}, z_{A}\right)$ satisfies the following equations:

where $\left(x_{o}, y_{o}, z_{o}\right)$ is the coordinate of the growing central point, $\left(x_{A}, y_{A}, z_{A}\right)$ is the coordinate of cement particle voxel after first rotation and $\left(x_{A^{\prime}}, y_{A}{ }^{\prime \prime}, z_{A}{ }^{\prime \prime}\right)$ is the final coordinate after second rotation.

A typical example of a $2 \mathrm{D}$ irregular particle rotated by $30^{\circ}$ is shown in Fig. 2. It can be seen that a few holes like "sieve" emerge in the interior of this particle after the rotation. This can be ascribed to the overlapping of some pixels after being rotated by a certain angle. In fact, this "sieve hole" phenomenon is very common in discrete-based packing model [16].

Step IV: These existing "sieve holes" can not only result in particle defects, but decrease particle volume stability. In order to eliminate "sieve holes", Boolean operations are used herein following the detailed processing procedure: first of all, the empty space outside of the particle is lightened (black region in Fig. 2). Subsequently, the remaining empty space pixels are converted into particle pixels. Finally, the lightened empty space pixels are turned back into initial status. Through this transformation, an intact particle rotated by a random angle can be obtained. It should be mentioned that such implementation may lead to some errors in a few pixels, while the number 
of these pixels can be negligible compared to the total number of pixels used to represent a particle that is usually in hundreds to houndreds of thousands in 3D.

Step V: Once the individual particle with a random orientation is reconstructed in the growing space, the next step is to throw this particle into RVE of cement mixes. In this work, the widely used random placement algorithm is chosen and implemented to rebuild packing of irregular-shaped cement particles. Furthermore, periodic boundary conditions are used at faces and edges of RVE in order to minimise finite size effects. During particle packing, if a newly introduced particle does not overlap with others, it is allowed to stay; otherwise, another position should be tried. The intersection and overlap of particles are evaluated through checking whether the particles have shared pixels. In order to increase packing efficiency and achieve a higher packing density in the RVE, the minimum circumscribed circle (MCC) method as shown in Fig. 2 is employed to assess the judging regions between irregular-shaped particles. For discrete irregular-shaped particles, the diameter of MCC can be easily determined by searching the largest distance between pixels on particle surface.

The Steps I to V are repeated until all desired particles are placed into the RVE. It should be pointed out that the particle packing starts with the largest particles and then smaller particles so that the remaining available space can then be filled by even smaller particles and a higher packing density can be achieved.

\section{Cement hydration model}

In this work, the cement hydration model CEMHYD3D is used to incorporate the irregular-shaped cement particles and simulate the subsequent hydration process, which consists of two main steps, i.e., cement particle segmentation and cement hydration process. 


\subsection{Cement particle segmentation}

In addition to irregular-shaped cement particle packing, the mineral phases on generated cement particles in RVE should be determined. Herein, the autocorrelation function (ACF) method is used to assign four primary mineral phases (i.e., $\mathrm{C}_{3} \mathrm{~S}, \mathrm{C}_{2} \mathrm{~S}, \mathrm{C}_{3} \mathrm{~A}$ and $\mathrm{C}_{4} \mathrm{AF}$ ) to each particle in RVE based on the measured volume fractions, surface area fractions and spatial distribution of these four mineral phases in cement powders by scanning electron microscopy (SEM) along with mapping technique. The sample preparation and image processing are described in detail in Section 4.1. First of all, the two-point correlation function for silicate phases (i.e., $\mathrm{C}_{3} \mathrm{~S}$ and $\mathrm{C}_{2} \mathrm{~S}$ ) obtained from the segmented SEM image is employed to separate the 3D irregular-shaped cement particles into silicate phases and aluminate phases (i.e., $\mathrm{C}_{3} \mathrm{~A}$ and $\mathrm{C}_{4} \mathrm{AF}$ ) through assigning random numbers with a normal distribution to the voxels in RVE using the Box-Muller method [33] in combination with stereology rules [34] and sintering algorithm [35]. Subsequently, the silicate phases are further divided into $C_{3} S$ and $C_{2} S$ based on $A C F$ of $C_{3} S$, while the aluminate phases are segmented into $C_{3} A$ and $\mathrm{C}_{4} \mathrm{AF}$ based on $\mathrm{ACF}$ of $\mathrm{C}_{3} \mathrm{~A}$. Therefore, the microstructure of pre-hydration cement paste consisting of four mineral phases can be obtained. The readers are referred to $[13,36]$ for more details about segmentation of cement particles.

\subsection{Cement hydration process}

After obtaining the 3D microstructure of pre-hydration cement paste with irregular-shaped cement particles and a desired w/c ratio, the evolution rules for modelling hydration process should be defined. In this work, the evolution rules given in $[13,14]$ that were developed on the basis of cellular automation theory and random walk algorithms are applied using CEMHYD3D model. The hydration evolution is simulated via an iterative process consisting of discrete cycles. In each cycle, through manipulating dissolution, diffusion and precipitation of different phase voxels based on 
probabilities belonging to each voxel, the hydration reaction and microstructural development are continuously implemented. Basically, the relevant voxels to different mineral phases dissolve from the surface of cement particles and diffuse in the space available up to a certain number of steps and then react to form hydration product voxels due to nucleation or collision with other phase voxels. In order to relate the time-dependent hydration process to real time, the relationship between computational cycles $(n)$ and age $(t)$ of the cement paste is controlled by parabolic hydration kinetics meets the following equation [13]:

$$
t=\beta n^{2}
$$

where $\beta$ is the conversion factor that can be further calibrated with real cement hydration properties, i.e., hydration heat and chemical shrinkage. Once $\beta$ is determined, the detailed information of cement hydration process and microstructural development can be continuously acquired.

\section{Experiment and modelling}

\subsection{Experimental programme}

In order to determine input parameters of raw materials for simulations and validate simulations, some experiments were performed.

\subsubsection{Materials and sample preparation}

A Chinese cement named P. I Portland cement (similar to ASTM Type I Portland cement) with weight percentage of $5 \%$ gypsum was used to prepare the cement paste samples. The chemical composition of cement is given in Table 1 . The average density of cement is $3.15 \mathrm{~g} / \mathrm{cm}^{3}$. The PSD (equivalent spherical diameter) of cement was measured using laser particle size analyzer (Microtrac S3500) and its according specific surface area is $465.8 \mathrm{~m}^{2} / \mathrm{kg}$. The initial and final setting times of cement paste at a w/c ratio of 0.274 with normal consistency measured by Vicat needle method [37] are $132 \mathrm{~min}$ and $187 \mathrm{~min}$, respectively. In terms of mix proportions, three 
cement pastes with w/c ratios of $0.23,0.35$ and 0.53 were designed. The specimens were cast in 100 $\times 300 \mathrm{~mm}$ cylinders and demoulded after $24 \mathrm{~h}$. Afterwards, the specimens were cured in a standard curing room with a relative humidity of $95 \%$ and temperature of $20{ }^{\circ} \mathrm{C}$ until testing.

\subsubsection{Mineral phase distributions in cement powders}

To determine the spatial distribution of four primary mineral phases in real cement powders, SEM along with mapping technique were used to obtain high-quality images. Furthermore, the ACF of each mineral phase in cement powders for segmenting reconstructed irregular-shaped particles in 3D needs to be determined from the segmented SEM image using image analysis technique. The details about the corresponding experimental procedure and image processing can be found in our previous paper [38] and Refs. [39, 40].

The cement powders were mixed with low-viscosity epoxy resin (cement-to-epoxy resin ratio $=$ 1:3 in mass) and then filled in several cylindrical moulds with diameter of $30 \mathrm{~mm}$ under vacuum. Afterwards, they were cured in drying oven at $45{ }^{\circ} \mathrm{C}$ for at least $6 \mathrm{~h}$. The demoulded specimens were polished with sandpaper 600,800 and 1200 grit, followed by 9,3 and $0.25 \mu \mathrm{m}$ diamond paste on an automatic polishing machine (Buehler Phoenix 4000). Each polishing step with sandpaper and diamond paste lasted $2 \mathrm{~min}$ and $30 \mathrm{~min}$, respectively. The polished specimens were then coated with a 15-nm thick carbon coating. After that, the prepared specimens were placed into the environment scanning electron microscope chamber with 1 Torr pressure and accelerating voltage of the beamer of $15 \mathrm{kV}$. Several representative background electron images (BEI) with different magnification levels were taken at random locations. The higher atomic number of mineral phase has, the greater grey level is. In order to better distinguish different mineral phases based on grey levels, a filter process is required to reduce the noise or imperfections of image. In this work, the medium grey level of a filter composed of $3 \times 3$ pixels was used to replace the grey level of central 
pixel, which was found to be also able to preserve strong contrast variations [41]. With such process, the mineral phases with large difference in grey level (or atomic number) can be obviously identified. However, it is still difficult to distinguish mineral phases with similar grey levels, e.g., $\mathrm{C}_{2} \mathrm{~S}$ and $\mathrm{C}_{3} \mathrm{~A}$. As such, additional process such as $\mathrm{X}$-ray spectroscopy mapping using different elements including $\mathrm{Ca}, \mathrm{Si}, \mathrm{Al}$, and $\mathrm{Fe}$ is needed for further segmentation and identify these phases in BEI. An image magnification level of 800 was found to be acceptable in order to get a stable mineral phase distribution of cement powders based on a series of statistical analysis [42]. Therefore, such magnification level was used in this work.

Based on the above-mentioned procedures, the cement particles in BEI can be divided into four mineral phases. Fig. 7 shows an example of the segmented image of cement particles with size of $512 \times 400$ pixels. The volume fraction and surface area of each mineral phase can be determined through point-by-point scanning along the area and perimeter in the segmented image based on stereoscopy theory [43]. The measured volume fraction and surface area were compared with the theoretical values calculated using Bogue equation in order to assess the accuracy of statistical analysis, which is shown in Table 2. It can be seen that the measured and theoretical volume fractions of four mineral phases are close to each other, which indicates that the segmented image can accurately represent the distribution of mineral phases in real cement particles.

Based on the segmented image of cement powder, the ACFs of silicate phase (i.e., $\mathrm{C}_{3} \mathrm{~S}+\mathrm{C}_{2} \mathrm{~S}$ ), $\mathrm{C}_{2} \mathrm{~S}$ and $\mathrm{C}_{3} \mathrm{~A}$, can be determined following the methods mentioned in Section 3.1. The normalized autocorrelation of $\mathrm{C}_{3} \mathrm{~S}+\mathrm{C}_{2} \mathrm{~S}, \mathrm{C}_{3} \mathrm{~S}$ and $\mathrm{C}_{3} \mathrm{~A}$ against distance of image is plotted in Fig. 8. It can be seen that all ACFs of cement dramatically decrease in the first 5-pixel region but reach steady values as the distance increases. The ACF of silicate phase is still around 0.37 after 5-pixel distance, while the $\mathrm{ACF}$ of $\mathrm{C}_{3} \mathrm{~A}$ is less than 0.05 , which implies that the $\mathrm{C}_{3} \mathrm{~S}$ and $\mathrm{C}_{2} \mathrm{~S}$ phases are composed of 
large clusters in cement particles, while the $\mathrm{C}_{3} \mathrm{~A}$ phase consists of numerous small local clusters.

\subsubsection{Hydration heat}

To validate the simulation of cement hydration process, the reaction heat of cement pastes with $\mathrm{w} / \mathrm{c}$ ratios of $0.23,0.35$ and 0.53 at $20^{\circ} \mathrm{C}$ was measured with an isothermal calorimetry. About $10 \mathrm{~g}$ paste of each mixture was used for measurement. The specific heat of cement paste $\left(C_{p}\right)$ can be expressed as:

$$
C_{\text {paste }}=C_{\text {cement }} x_{\text {cement }}+C_{\text {water }} x_{\text {water }}
$$

where $C_{c e m e n t}$ and $C_{\text {water }}$ are specific heat values of water and cement, and $x_{\text {cement }}$ and $x_{\text {water }}$ are mass fractions of water and cement in the mixture. Here, $C_{\text {cement }}=0.80 \mathrm{~J} /(\mathrm{g} \cdot \mathrm{K})$ and $C_{\text {water }}=4.18 \mathrm{~J} /(\mathrm{g} \cdot \mathrm{K})$ are adopted, which are similar to those given in [41].

\subsubsection{Degree of hydration}

The degree of hydration $(\mathrm{DoH})$ of cement paste specimens with w/c ratios $0.23,0.35$ and 0.53 was determined by mass loss on ignition between 105 and $950{ }^{\circ} \mathrm{C}$. The specimens were crushed at curing ages of 1, 7 and 28 days, respectively, and were then ground into powder using a mortar and pestle and flushed with absolute ethyl alcohol. The ground and flushed samples were placed into a $105^{\circ} \mathrm{C}$ vented oven overnight to eliminate the evaporable water. The powder with approximate $1 \mathrm{~g}$ was weighed (accurate to $0.0001 \mathrm{~g}$ ) and subsequently heated to $950{ }^{\circ} \mathrm{C}$ in a crucible for $4 \mathrm{~h}$. The non-evaporable water content $\left(W_{n}\right)$ can be calculated by [44]:

$$
W_{n}=\frac{m_{105^{\circ} \mathrm{C}}-m_{950^{\circ} \mathrm{C}}}{m_{950^{\circ} \mathrm{C}}}
$$

where $m_{105^{\circ} \mathrm{C}}$ and $m_{950^{\circ}} \mathrm{C}$ are the weights of powder at 105 and $950{ }^{\circ} \mathrm{C}$, respectively.

$$
\text { According to Power's model [45], the DoH can be determined as: }
$$

$$
\alpha_{c}=\frac{W_{n}}{0.23}-L_{c}
$$

where $\alpha_{c}$ and $L_{c}$ denote DoH and cement loss on ignition, respectively; 0.23 means that $1 \mathrm{~g}$ pure 
cement needs $0.23 \mathrm{~g}$ water for complete hydration.

\subsection{Modelling and simulation procedure}

The RVE size of cement paste was found to be $100 \times 100 \times 100 \mu \mathrm{m}^{3}[10,11,13]$. According to the real PSD, numerous cement particles with random orientations were randomly placed into a cubic RVE of $200 \times 200 \times 200$ voxels with a spatial resolution of $0.5 \mu \mathrm{m} / \mathrm{voxel}$ to produce initial microstructures of cement paste with w/c ratios of $0.23,0.35,0.53$ and 0.274 (the value for normal consistency). Most of the reconstructed cement particles can possess desired shapes at this spatial resolution. The random placement algorithm with minimum inscribed sphere method was employed for packing of cement particles with a desired density. During the packing process, some cement particles were stochasticly switched into gypsum particles in order to obtain a given volume fraction of gypsum. It is worth pointing out that those changeably-shaped small particles were not rotated in order to avoid volume errors. For achieving stable shapes, the number of constituent voxels of an individual cement particle was determined to be 300. In order to investigate the influence of particle shape on cement hydration process, a set of initial microstructures made up of similar cement particles with different eigenvectors were generated and used for simulations. For each w/c ratio, three RVEs were produced with spherical (DS), itermediate (M2) and elongated (M3) shaped cement particles (see Fig. 4), respectively. Using the obtained volume fractions, surface area fractions and ACFs of silicate phase, $\mathrm{C}_{3} \mathrm{~S}$ and $\mathrm{C}_{3} \mathrm{~A}$ from SEM images as shown in Table 2 and Fig. 8 , the segmentation of 3D irregular-shaped cement particles was carried out to divide cement particles into $\mathrm{C}_{3} \mathrm{~S}, \mathrm{C}_{2} \mathrm{~S}, \mathrm{C}_{3} \mathrm{~A}$ and $\mathrm{C}_{4} \mathrm{AF}$ in each RVE.

Based on the segmented initial microstructures, the hydration process of cement was subsequently simulated using the CEMHYD3D model. The corresponding hydration properties such as hydration heat, degree of hydration and setting behaviour were continuously determined. 
The specimens were assumed to be fully saturated and the curing temperature was $20^{\circ} \mathrm{C}$ for cement paste with w/c ratios of $0.23,0.35$ and 0.53 , and $23{ }^{\circ} \mathrm{C}$ for cement paste with w/c ratio of 0.274 , respectively. The simulation results were then compared with experimental data.

\section{Results and discussion}

\subsection{Initial 3D microstructure of cement paste}

\subsubsection{Geometric attributes}

Based on real PSD from laser particles analysis, three microstructures with different shapes at $\mathrm{w} / \mathrm{c}$ ratios of $0.23,0.35$ and 0.53 are reconstructed, using a random placement algorithm. A typical example of microstructures at at w/c the ratio of 0.35 is shown in Fig. 9 where red particles are cement clinkers and grey particles are gypsum. The multi-size particles from 0.549 to $44.764 \mu \mathrm{m}$, seen in Fig.11, are randomly thrown into RVE following the sequence from large ones to small ones. Meanwhile, the particles are allowed to contact with each other. Compared with the spherical-shaped initial microstructure, those microstructures consisting of cement and gypsum irregular particles with a certain shape are randomly located at the system without a certain orientation. In this resolution, the smallest particle that can be included by the digitization approach is 1 voxel in size of $0.5 \mu \mathrm{m}$. Although particles size are not smaller than $0.5 \mu \mathrm{m}$ in this study, however, for those particles with diameter smaller than $0.5 \mu \mathrm{m}$, if detected, these are regarded as one cubic clusters. This will result in the undesirable artifact because small particles contribute a significant amount of surface area though they represent a small volume fraction. The good approach for meshing finer cubes (using higher resolution) may overcome this drawback, while this accordingly results in lower calculating rate. Actually, this is not a significant puzzle, for sub-micrometer particles tend to flocculate into soft agglomerates during hydration process owe to Waals forces [46]. When the RVE is digitalized into $0.5 \mu \mathrm{m} / \mathrm{voxel}$, the volume of fine particles with 
size of smaller than $0.5 \mu \mathrm{m}$ accounts for pretty little. Additionally, the digitalized corase particles will increase surface area compared with those of smooth surface with the same volume. For example, the surface area of digitalized sphere with sufficient voxels is equal to 1.4 times of that of smooth sphere, seen in Fig. 5. Accordingly, this amplification of surface area will compensate for the loss of sub-micrometer particles' surface area.

In order to validate the random placement process of cement particles, the modeling PSD of cement mixes composed of spherical and irregular-shaped particles respectively at w/c ratios of $0.23,0.35$ and 0.53 is compared with the experimental data measured through laser diffraction technique, as shown in Fig. 10. Due to the discontinuous cubic size, the digitalized sphere is centered on the odd-integer diameter in dimension of bin size. It can be seen that the digitalized sphere diameters are not strictly consistent with those of equivalent spheres from experimental results and its modeling particle sizes tend to be large area. In addition, the curve of interval PSD of digitalized spherical cement particles fluctuates with particle diameter containing some peak points and shows no continuous changing tendency. By contrast, those interval and cumulative PSD curves of irregular-shaped particles with same measured diameters have good fitting with experimental curves, at least in the area of diameter lower than $35.0 \mu \mathrm{m}$. The interval curves of w/c ratios of 0.35 and 0.53 seem not correspond with real ones in large size area for insufficient particle volume, while these particles with diameter over $35.0 \mu \mathrm{m}$ only represent less than $3 \%$. In general, the modeling PSD of irregular-shaped cement particles has a realistic approximation of measured one.

Based on the measured volume fractions, surface area fractions and AFCs of mineral phases of cement particles, the homogeneous cement particles with different shapes in RVEs are segmented and the mineral phases are subsequently assigned to different voxels of cement particles. Thus, the initial microstructures of cement mixes containing respective shapes of cement particles with a 
given amount of mineral phases can be obtained. Fig. 11 shows the $2 \mathrm{D}$ slices of initial microstructures with spherical, intermediate and elongated shaped particles respectively extracted from the obtained 3D microstructures of pre-hydration cement with w/c ratio of 0.35 . It can be seen that each cement particle is made up of four different mineral phases and water is presented with saturated capillary pores in black. The gypsum is shown in grey colour. In comparison with 2D shapes of cement powders in SEM (see Fig. 7), the reconstructed particles comprised of irregular-shaped particles appear much more similar than those spherical particles, and some 2D shapes in the slices are almost identical to real ones. This implies that the generated RVE consisting

\subsubsection{Specific surface area}

Table 3 shows the determined specific surface areas of cement particles in RVEs of pre-hydration cement pastes with $\mathrm{w} / \mathrm{c}$ ratios of $0.23,0.35$ and 0.53 . The specific surface areas of cement particles increase with increasing $\mathrm{w} / \mathrm{c}$ ratio. This is ascribed to that more cement particles are placed into the RVEs in order to achieve a lower w/c ratio and cement particles tend to contact each other, especially for small particles. Accordingly, the specific surface area is reduced due to the interparticle contacts in RVE. The specific surface area is also related to the shape of cement particles. The specific surfae areas of RVEs composed of elongated-shaped particles at w/c ratios of 0.23, 0.35 and 0.53 are $482.6 \mathrm{~m}^{2} / \mathrm{kg}, 495.9 \mathrm{~m}^{2} / \mathrm{kg}$ and $503.7 \mathrm{~m}^{2} / \mathrm{kg}$, respectively, which are approximately $38 \%$ and $9 \%$ higher than those of RVEs consisting of spherical and intermediate shaped particles respectively at the same w/c ratio. This can be attributed to the similar contacts of self-similar particles.

Compared to measured specific surface area of $465.8 \mathrm{~m}^{2} / \mathrm{kg}$, the specific surface areas of RVEs made up of elongated-shaped cement particles are slightly (around 3-8\%) higher, while those of 
RVEs containing spherical particles are much smaller than experimental value; only $72.5 \%$ to $78.5 \%$ of experimental value. For intermediate-shaped systems, the specific surface areas tend to approach experimental one as the $\mathrm{w} / \mathrm{c}$ ratio increases and for $\mathrm{w} / \mathrm{c}=0.53$ and the simulated specific surface area is only about $1 \%$ higher than the measured one.

It should be noted that unavoidable underestimate of surface area in the discrete packing system may occur resulting from coarse cubes and coarse discretization of PSD. Using reasonable mix proportion of different shaped cement particles can compensate for the lost surface area and even be totally consisitent with the experimetal results. However, there seems to be no too much sense, because detailed shapes of small cement particles have not been totally determined until now. Normally, we can only employ surface area as the quantitative measure in cement powders. Furthermore, it has been shown that the shapes of cement particles from the same type of cement have somewhat self-similarity in 2D SEM and 3D XCT images [31]. Therefore, to model integrate particle kinetics including shaped discrepancy, as simulated cement packing system consisting of one certain shaped particles demonstrates same surface area as real cement particles, its hydration process can be treated as the equivalent of the real one containing multi-shaped particles for simplification. In this work, the intermediate-shaped particles can be considered as equvalent particles of Type I Portland cement.

\subsection{D microstructure of hydrating cement paste}

\subsubsection{Hydration heat}

Noticeably, for comparison of the effects of shaped discrepancy of cement particles, there are two assumptions for simulated microstructural evolution of cement pastes. In the early age for cement pastes, the surface area of initial cement particles is the dominant factor to influence hydration process of cement paste [47]. Thus, as mentioned in Section 5.1.2, the first assumption is 
that simulated microstructure generated using one certain shaped particles with the same surface area as the real cement can represent real cement hydration process. In this study, the simulated hydration process of intermediate-shaped cement particles is calibrated using the real hydration process of Type I cement. Furthermore, in the initial CEMHYD3D model using digitalized spheres as cement particles, the value of conversion factor $\beta$ is close to a constant value in cement pastes at different w/c ratios [13] or with different cement fineness [48]. More importantly, the experimental validation of hydration preocess in simulated cement pastes is impossible to be obtained for specific shaped particles. Therefore, $\beta$ is subsequently supposed to be a constant value for all simulations with different shaped particles. Although conversion factor $\beta$ s in different microstructures generated using different shaped cement particles are various as calibrated with the real experiment data, the surface area difference of cement particles is not yet included without comparison of the effects on hydration kinetics. Meanwhile, the meaning of conversion factor $\beta$ in Eq. (15) has no rational interpretation until now. As such, these assumptions are reasonable as considering the effect of surface area of particles on cement hydration.

Based on the experimental data of hydration heat in cement pastes at the w/c ratio of 0.35 and Eq. 15, the factor $\beta$ for the equivalent microstructures of a mixture of Type I Portland cement and water reconstructured using intermediate-shaped particles can be calculated to be $0.000096 \mathrm{~h} / \mathrm{cycle}^{2}$. Fig. 12 shows a comparsion of the simulated and experimental hydration heat of cement pastes generated using different shaped cement particles with w/c ratios of $0.23,0.35$ and 0.53 . The curves of hydration heat against curing time for intermediate-shaped cement pastes are in good agreement with experimental results for all $\mathrm{w} / \mathrm{c}$ ratios. This indicates that for irregular-shaped cement paste the hydration rate is insensitive to $\mathrm{w} / \mathrm{c}$ ratio, which is consistent with the finding presented in [2, 13]. Moverover, it in turn validates that the assumption of constant value for $\beta$ in cement pastes at 
different $\mathrm{w} / \mathrm{c}$ ratios is reasonable.

The hydration heat of irregular-shaped system in the early period up to $60 \mathrm{~h}$ is much higher than that of spherical system regardless of w/c ratios, which can be ascribed to the corresponding larger specific surface area (see Table 3). The released heat of spherical system at $60 \mathrm{~h}$ is approximately $80 \%$ of that of intermediate-shaped system, while the released heat of elongated-shaped system is slightly higher to that of intermediate-shaped system. As w/c ratio increases, the difference in hydration rate of these three systems increases. In addition, the hydration rate of each system increases with increasing w/c ratio, while the increase in hydration rate of spherical system is less obvious due to the smaller specific surface area. This implies that the equiaxed cement particles with similar chemical composition and PSD can contribute to the reduction in heat release rate. The cement particle shapes can be carefully designed during the manufacture process in order to extend the heat release process and thus limit the development of thermal stress and minimise the heat induced cracking in cementitious materials.

\subsubsection{Degree of hydration}

The simulated DoH of cement pastes with spherical, intermediate and elongated shaped particles and w/c ratios of $0.23,0.35$ and 0.53 as a function of curing time is plotted in Fig. 13 together with experimental data. As can be seen that the simulated DoH of intermediate-shaped system fits very well with the measured DoH based on non-evaporable water content [41]. It implies that $\beta=0.000096 \mathrm{~h} /$ cycle $^{2}$ for intermediate-shaped cement particles is rational. The DoH of elongated-shaped system is the greatest, followed by intermediate-shaped system, while the DoH of spherical system is the lowest for all w/c ratios. This can be attributed to the difference in their surface areas. The elongated-shaped particles have the largest surface area, while the surface area of spherical particles is the smallest. In the early period up to $6 \mathrm{~h}$, the DoHs of elongated-shaped 
system with $\mathrm{w} / \mathrm{c}$ ratios of $0.23,0.35$ and 0.53 are $0.15,0,18$ and 0.21 respectively, which are around 5 times those of spherical system. At 28 d (i.e., 672 h), the DoHs of systems with different particle shapes for w/c ratios of 0.23 and 0.35 tend to be similar to each other and reach 0.45 at w/c $=0.23$ and 0.64 at $\mathrm{w} / \mathrm{c}=0.35$, respectively.

Fig. 14 shows the simulated 3D microstructures and corresponding 2D slices of hydrating cement pastes composed of spherical, intermediate and elongated shaped cement particles with w/c ratio of 0.35 at $6 \mathrm{~h}$. It can be seen that during the first $6 \mathrm{~h}$ of hydration, the small particles and the parts of sharp surfaces on irregular-shaped large cement particles graduately disappear as cement particles dissolve and hydration products form in capillary pores around original cement particles.

The high reaction rate in irregular-shaped system can be attributed to the local sharp surface regions, which is consistent with that shown in [49] that a cement particle with more parts of high positive curvature has a higher reaction rate due to more dissoluble surface as compared to that with lower or negative curvature. Additionally, the hydration process of cement particles is simulated according to the dissolution-diffusion-nucleation-controlled mechanism using the CEMHYD3D model [50]. The hydration products such as $\mathrm{C}-\mathrm{S}-\mathrm{H}$ and $\mathrm{CH}$ are produced as a result of collision of dissoluble phase clusters with existing solid-phase surface. Compared to spherical cement particles, the additional surface area and roughness of irregular-shaped cement particles can lead to an increase in nucleation positions and further contribute to higher collision probability. Consequently, the cement particle shapes can noticeably affect cement hydration process and microstructural formation of cement paste in the early period.

As seen in the $28 \mathrm{~d}$ microstructures of cement pastes in Fig. 14, the initial shapes of cement particles cannot be clearly distinguished and the simulated complex microstructures of cement pastes look similar to each other. At $28 \mathrm{~d}$ of hydration, cement pastes with different shapes have 
641 similar DoH at $w / c=0.35$. At the same time, the surfaces of unhydrated cement particles in any

642 shaped systems all tend to become more spherical with time, which implies that any discrepancies

643

644

645

646

647

648

649

650

651

652

653

654

655

656

657

658

659

660

661

662

663

resulting from cement particle shape during hydration process would be reduced as hydration proceeds. Such tendency becomes more obvious as the $\mathrm{w} / \mathrm{c}$ ratio increases. At $\mathrm{w} / \mathrm{c}=0.53$, the ultimate DoH of elongated-shaped cement paste is found to be 0.86 at 90 days, which is around 13\% higher than that of spherical system, which can be ascribed to the relatively higher surface area of elongated-shaped particles. The elongated-shaped particles have thinner hydration products around unhydrated cement particles when having same DoH and the thickness of hydration products along the orientation of $b-c$ plane for irregular-shaped particles is the narrowest according to theoretical analysis if assuming a homogeneous distribution of mineral phases of cement. The distance of water penetrating through hydration products into surface of unhydrated cement particle in RVE with larger particle surface area is narrower than that a with smaller one. As a consequence, water in capillary pores can relatively easily pass through the barrier layer in elongated-shaped system if there is sufficient water, which can promote further cement hydration.

\subsubsection{Connectivity of solid phases}

Numerous studies have shown that the setting behaviour of cement paste measured by Vicat needle method correlates with the connectivity of solid phases based on the numberical simulations considering spherical cement particles $[2,42,51]$. In order to investigate the effect of cement particle shape on percolation threshold of solid phases and the relevant relationship between setting time and percolation of solid phases, the connectivity of solid phases in cement pastes with different particle shapes at $\mathrm{w} / \mathrm{c}$ ratios of 0.274 (normal consistency) and 0.53 as a function of curing time is simulated and presented in Fig. 15 together with experimental setting time of cement.

It can be seen that the shape of cement particle has a significant influence on percolation 
664

665

666

667

668

669

670

671

672

673

674

675

676

677

678

679

680

681

682

683

684

threshold of solid phases in cement paste. The corresponding hyration time to percolation threshold of solid phases for irregular-shaped systems is lower than that for spherical system due to the large surface area of cement particles. The difference in connectivity of solid phases between the systems increases with increasing $\mathrm{w} / \mathrm{c}$ ratio. At $\mathrm{w} / \mathrm{c}=0.274$, the percolation of solid phases for spherical system occurs at around $50 \mathrm{~min}$ of hydration that is approximately $15 \mathrm{~min}$ later than that for elongated-shaped system, while the time discrepancy of reaching solid percolation threshold for spherical and elongated-shaped systems at $\mathrm{w} / \mathrm{c}=0.53$ is 4.5 h, i.e., $270 \mathrm{~min}$ (see Fig. 15b).

In comparison with the experimental initial and final setting times of $132 \mathrm{~min}$ and $187 \mathrm{~min}$ respectively, the percolation of solid phases in three systems all takes place prior to the initial setting, which is consistent with Refs. [2, 51]. With respect to the connectivity of solid phases between initial and final setting, they are found to be close to each other for three systems, between 0.88 and 0.97 , which is in good agreement with the finding shown in [2] that the connectivity values of solid phases between initial and final setting were 0.80 and 0.92 respectively for CCRL-116 using reconstructed cement particles from XCT. However, Haecker et al. [51] reported that the connectivity values of solid phases between initial and final setting were found to be 0.4 and 0.75 respectively. An image resolution of $1 \mu \mathrm{m} /$ voxel was used in these two studies. In fact, the percolation threshold is highly dependent on the used image resolution. The higher the resolution, the lower solid percolation threshold the specimens had [22]. On the other hand, a specific concern regarding setting time determined using Vicat method is somewhat arbitrarily defined and may not correspond to a specific aspect of microstructure formation of cement paste [52]. As such, the predicted percolation of solid phases may not be strictly consistent with setting behaviour measured using this technique.

In order to estimate the influence of particle shapes on percolation of solid phases regardless of 
kinetics of cement hydration, the connectivity of solid phases is plotted as a function of DoH in Fig. 16. At a lower $\mathrm{w} / \mathrm{c}$ ratio (i.e., $\mathrm{w} / \mathrm{c}=0.274$ ), the solid percolation curves are quite similar to each other for microstructures of hydrating cement paste with various cement particle shapes and the solid percolation thresholds are found to be around $0.007 \mathrm{DoH}$. In contrast, the solid percolation threshold of microstructures of $\mathrm{w} / \mathrm{c}=0.53$ cement paste with spherical particles happens at 0.035 DoH, which is approximately two times that of elongated-shaped system. Due to geometric attributes, the elongated-shaped particles in the initial microstructure tend to interconnect with each other, which accordingly results in a lower solid-to-solid spacing [16]. Compared to microstructures of cement paste with lower w/c ratios, more hydration products are required to form a percolation network of solid phases in the microstructures with a higher w/c ratio due to the larger solid-to-solid spacing. Based on the recently developed algorithm (see [28] for details), the solid-to-solid spacing in RVEs of cement mixes with w/c ratios of 0.274 and 0.53 can be determined, which is shown in

Fig. 17. It can be seen that the average solid-to-solid spacing in RVEs of cement mixes with w/c ratio of 0.53 is about $45 \%$ larger than that of $w / c=0.23$ cement mixes regardless of cement particle shapes. In addition, it is indicated that the solid-to-solid spacing in RVEs with spherical particles is approximately $20 \%$ larger than that in RVEs with elongated-shaped particles at same w/c ratios, as a result of which the solid percolation threshold of RVEs with spherical particles is higher. This is in good consistence with the conclusion drawn by Garboczi et al. [53] for overlapping ellipsoids that the solid percolation threshold increased significantly as particles became more equiaxed.

\section{Conclusions}

In this paper, an integrated modelling approach consisting of central growth model based on discrete method and cellular automation, particle packing algorithm and cement hydration model is presented to estimate the influence of cement particle shape on cement hydration and 
microstructural characteristics of hydrating cement paste. Firstly, a series of cement particles with

regular (i.e., sphere) and irregular shapes are generated using central growth model. Subsequently, the representative volume elements of real Portland cement mixes with different $w / c$ ratios (i.e., initial 3D microstructures) are produced using the proposed packing procedure on the basis of measured particle size distribution of cement powders. Finally, the CEMHYD3D model is used to simulate cement hydration process and microstructural formation. The influence of cement particle shape on hydration heat, degree of hydration and percolation of solid phases is then investigated in a quantitative manner. Based on the findings of this study, the following conclusions can be drawn:

- The proposed central growth model can well reconstruct irregular-shaped cement particles. The generated particles are not only shape-random from the perspective of morphology but shape-controllable in terms of classification on the condition of sufficient constituent elements. Two shape descriptors, i.e., sphericity and normalized semi-axes lengths of equivalent inertia ellipsoid, can be used as criteria to generate unique irregular-shaped particles and create the corresponding shape library of particles. Compared to vector-based method, the discrete method employed in this study can easily determine particle geometric attributes and effectively achieve desired packing density.

- The simulated particle size distribution and specific surface areas of initial microstructures made up of irregular-shaped cement particles are in good agreement with experimental data regardless of w/c ratios, while a large discrepancy exists between those for regular-shaped (i.e., spherical) cement particles and experimental data. In addition, the reconstructed particles comprised of irregular-shaped particles appear much more similar than those spherical particles, which indicates that the generated representative volume elements consisting of irregular-shaped particles can better represent the real pre-hydration cement paste. 
- The shape of cement particles has a significance influence on hydration heat, degree of hydration and percolation of solid phases in hydrating cement paste, which is mainly due to the difference in specific surface area between regular-shaped (e.g., spherical) particles and irregular-shaped (e.g., intermediate and elongated-shaped) particles. Moreover, the less observed geometric effect of cement particles is estimated to influence hydration process and final degree of hydration (DoH), while this effect will be enhanced with water-to-cement ratio increasing. Among these three microstructures, the formation of a percolating network in the microstructure with geometry of more non-equiaxed shape occurs at the lower DoH. At the lower water-to-cement ratio, large padding solid fraction can significantly lessen particle geometry effect on setting behaviour.

\section{Acknowledgements}

The financial support from National Natural Science Foundation of China (No. 51378116, No. 51678143 and No. 51408597), Ministry of Science and Technology of China (973 Program, No. 2015CB655102), China Scholarship Council (CSC) and The Royal Society (IE150587) are gratefully acknowledged. The authors also would like to thank Dr. Dale Bentz and Dr. Edward Garboczi from the National Institute for Standards and Testing, USA, for kindly providing the source codes of CEMHYD3D.

\section{References}

[1] S.J. Blott, K. Pye, Particle shape: a review and new methods of characterization and classification, Sedimentology, 55 (2008) 31-63.

[2] J.W. Bullard, E.J. Garboczi, A model investigation of the influence of particle shape on portland cement hydration, Cement and Concrete Research, 36 (2006) 1007-1015.

[3] S. Jamkar, C. Rao, Index of aggregate particle shape and texture of coarse aggregate as a 
parameter for concrete mix proportioning, Cement and Concrete Research, 34 (2004) 2021-2027.

[4] M.R. Geiker, M. Brandl, L.N. Thrane, L.F. Nielsen, On the effect of coarse aggregate fraction and shape on the rheological properties of self-compacting concrete, Cement, concrete and aggregates, 24 (2002) 3-6.

[5] L. Liu, D. Shen, H. Chen, W. Xu, Aggregate shape effect on the diffusivity of mortar: a 3D numerical investigation by random packing models of ellipsoidal particles and of convex polyhedral particles, Computers \& Structures, 144 (2014) 40-51.

[6] E.J. Garboczi, G.S. Cheok, W.C. Stone, Using LADAR to characterize the 3-D shape of aggregates: Preliminary results, Cement and Concrete Research, 36 (2006) 1072-1075.

[7] J.-P. Latham, A. Munjiza, X. Garcia, J. Xiang, R. Guises, Three-dimensional particle shape acquisition and use of shape library for DEM and FEM/DEM simulation, Minerals Engineering, 21 (2008) 797-805.

[8] E.J. Garboczi, Three-dimensional mathematical analysis of particle shape using X-ray tomography and spherical harmonics: Application to aggregates used in concrete, Cement and concrete research, 32 (2002) 1621-1638.

[9] E.J. Garboczi, J.W. Bullard, Shape analysis of a reference cement, Cement and Concrete Research, 34 (2004) 1933-1937.

[10] K. Van Breugel, Numerical simulation of hydration and microstructural development in hardening cement-based materials (I) theory, Cement and Concrete Research, 25 (1995) 319-331.

[11] S. Bishnoi, K.L. Scrivener, $\mu$ ic: A new platform for modelling the hydration of cements, Cement and Concrete Research, 39 (2009) 266-274.

[12] K. Maekawa, T. Ishida, T. Kishi, Multi-scale modeling of concrete performance, Journal of Advanced Concrete Technology, 1 (2003) 91-126. 
[13] D.P. Bentz, Three - Dimensional Computer Simulation of Portland Cement Hydration and Microstructure Development, Journal of the American Ceramic Society, 80 (1997) 3-21.

[14] D.P. Bentz, CEMHYD3D: A three-dimensional cement hydration and microstructure development modelling package. Version 2.0, National Institute of Standards and Technology Interagency Report, 7232 (2000).

[15] D.P. Bentz, Quantitative comparison of real and CEMHYD3D model microstructures using correlation functions, Cement and Concrete Research, 36 (2006) 259-263.

[16] X. Jia, R. Williams, A packing algorithm for particles of arbitrary shapes, Powder technology, $120(2001)$ 175-186.

[17] P.A. Cundall, Formulation of a three-dimensional distinct element model—Part I. A scheme to detect and represent contacts in a system composed of many polyhedral blocks, International Journal of Rock Mechanics and Mining Sciences \& Geomechanics Abstracts, Elsevier, 1988, pp. 107-116.

[18] E.G. Nezami, Y.M. Hashash, D. Zhao, J. Ghaboussi, A fast contact detection algorithm for 3-D discrete element method, Computers and geotechnics, 31 (2004) 575-587.

[19] L. Liu, W. Sun, G. Ye, H. Chen, Z. Qian, Estimation of the ionic diffusivity of virtual cement paste by random walk algorithm, Construction and Building Materials, 28 (2012) 405-413.

[20] M. Zhang, G. Ye, K. van Breugel, Modeling of ionic diffusivity in non-saturated cement-based materials using lattice Boltzmann method, Cement and Concrete Research, 42 (2012) 1524-1533.

[21] S. Wolfram, Theory and applications of cellular automata, World scientific Singapore1986.

[22] E.J. Garboczi, D.P. Bentz, The effect of statistical fluctuation, finite size error, and digital resolution on the phase percolation and transport properties of the NIST cement hydration model, Cement and Concrete Research, 31 (2001) 1501-1514. 
[23] J.C. Russ, J.R. Matey, A.J. Mallinckrodt, S. McKay, The image processing handbook, Computers in Physics, 8 (1994) 177-178.

[24] J.W. Bullard, E.J. Garboczi, Defining shape measures for 3D star-shaped particles: sphericity, roundness, and dimensions, Powder technology, 249 (2013) 241-252.

[25] M.A. Taylor, E.J. Garboczi, S. Erdogan, D. Fowler, Some properties of irregular 3-D particles, Powder Technology, 162 (2006) 1-15.

[26] M.A. Taylor, Quantitative measures for shape and size of particles, Powder Technology, 124 (2002) 94-100.

[27] H. Wadell, Volume, shape, and roundness of rock particles, The Journal of Geology, 40 (1932) 443-451.

[28] Y. Zhang, C. Liu, Z. Liu, G. Liu, L. Yang, Modelling of diffusion behavior of ions in low-density and high-density calcium silicate hydrate, Construction and Building Materials, 155 (2017) 965-980.

[29] P. Campbell, S. Abhyankar, Fractals, form, chance and dimension, Springer, 1978.

[30] C.M. Cooper, S. Testa, A quick method of determining rock surface area for quantification of the invertebrate community, Hydrobiologia, 452 (2001) 203-208.

[31] L. Holzer, R.J. Flatt, S.T. Erdoğan, J.W. Bullard, E.J. Garboczi, Shape comparison between 0.4-2.0 and 20-60 $\mu \mathrm{m}$ cement particles, Journal of the American Ceramic Society, 93 (2010) 1626-1633.

[32] V.I. Arnol'd, Mathematical methods of classical mechanics, Springer Science \& Business Media2013.

[33] A.M. Law, W.D. Kelton, W.D. Kelton, Simulation modeling and analysis, McGraw-Hill New York1991. 
[34] J.C. Russ, R.T. Dehoff, Practical stereology, Springer Science \& Business Media2012.

[35] D. Bentz, P. Pimienta, E. Garboczi, W. Carter, Cellular Automaton Simulations of Surface Mass Transport Due to Curvature Gradients: Simulations of Sintering in 3-D, MRS Proceedings, Cambridge Univ Press, 1991, pp. 413.

[36] D.P. Bentz, N.S. Martys, Hydraulic radius and transport in reconstructed model three-dimensional porous media, Transport in porous media, 17 (1994) 221-238.

[37] C. ASTM, 191. Test Method for Time of Setting of Hydraulic Cement by Vicat Needle, Annual Book of ASTM Standards, (2004).

[38] C. Liu, Y. Zhang, Z. Liu, M. Zhang, Experimental and modelling investigation of cement hydration and microstructural development with fly ash (in preparation).

[39] P. Stutzman, Scanning electron microscopy imaging of hydraulic cement microstructure, Cement and Concrete Composites, 26 (2004) 957-966.

[40] D.P. Bentz, A three-dimensional cement hydration and microstructure program: I. hydration rate, heat of hydration, and chemical shrinkage, Building and Fire Research Laboratory, National Institute of Technology1995.

[41] V. Kocaba, E. Gallucci, K.L. Scrivener, Methods for determination of degree of reaction of slag in blended cement pastes, Cement and Concrete Research, 42 (2012) 511-525.

[42] Z. Liu, Y. Zhang, Q. Jiang, W. Zhang, J. Wu, Solid phases percolation and capillary pores depercolation in hydrating cement pastes, Journal of Materials in Civil Engineering, 26 (2013) 04014090.

[43] C. Liu, Y. Zhang, Z. Liu, G. Liu, L. Yang, A numerical method for investigating ion diffusion behavior in low-density and high-density calcium silicate hydrate, submitted to Construction and Building Materials. 
[44] J. Escalante-Garcia, Nonevaporable water from neat OPC and replacement materials in composite cements hydrated at different temperatures, Cement and Concrete Research, 33 (2003) 1883-1888.

[45] T.C. Powers, The Nonevaporable Water Content of Hardened Portland-Cement Paste--Its Significance for Concrete Research and Its Method of Determination, 1900.

[46] M. Yang, C. Neubauer, H. Jennings, Interparticle potential and sedimentation behavior of cement suspensions: Review and results from paste, Advanced Cement Based Materials, 5 (1997) $1-7$.

[47] D.P. Bentz, G. Sant, J. Weiss, Early-age properties of cement-based materials. I: Influence of cement fineness, Journal of materials in civil engineering, 20 (2008) 502-508.

[48] D.P. Bentz, E.J. Garboczi, C.J. Haecker, O.M. Jensen, Effects of cement particle size distribution on performance properties of Portland cement-based materials, Cement and Concrete Research, 29 (1999) 1663-1671.

[49] J.W. Bullard, Digital-image-based models of two-dimensional microstructural evolution by surface diffusion and vapor transport, Journal of applied physics, 81 (1997) 159-168.

[50] J.W. Bullard, H.M. Jennings, R.A. Livingston, A. Nonat, G.W. Scherer, J.S. Schweitzer, K.L. Scrivener, J.J. Thomas, Mechanisms of cement hydration, Cement and Concrete Research, 41 (2011) 1208-1223.

[51] C. Haecker, D. Bentz, X. Feng, P. Stutzman, Prediction of cement physical properties by virtual testing, Cement International, 1 (2003) 86-92.

[52] J. Zhang, E.A. Weissinger, S. Peethamparan, G.W. Scherer, Early hydration and setting of oil well cement, Cement and Concrete research, 40 (2010) 1023-1033.

[53] E. Garboczi, K. Snyder, J. Douglas, M. Thorpe, Geometrical percolation threshold of 
overlapping ellipsoids, Physical review E, 52 (1995) 819.

872 


\section{Figures}

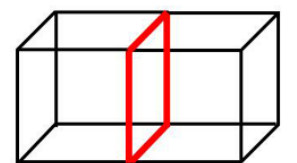

(a)

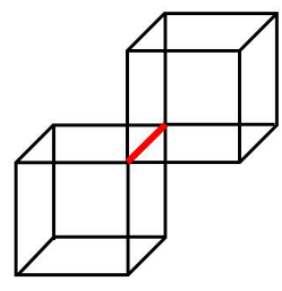

(b)

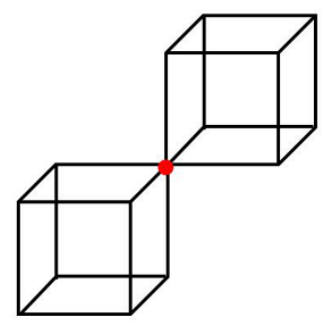

(c)

Fig. 1 Three relationships between adjacent voxels: (a) face-to-face neighbour model; (b) edge-toedge neighbour model; (c) point-to-point neighbour model
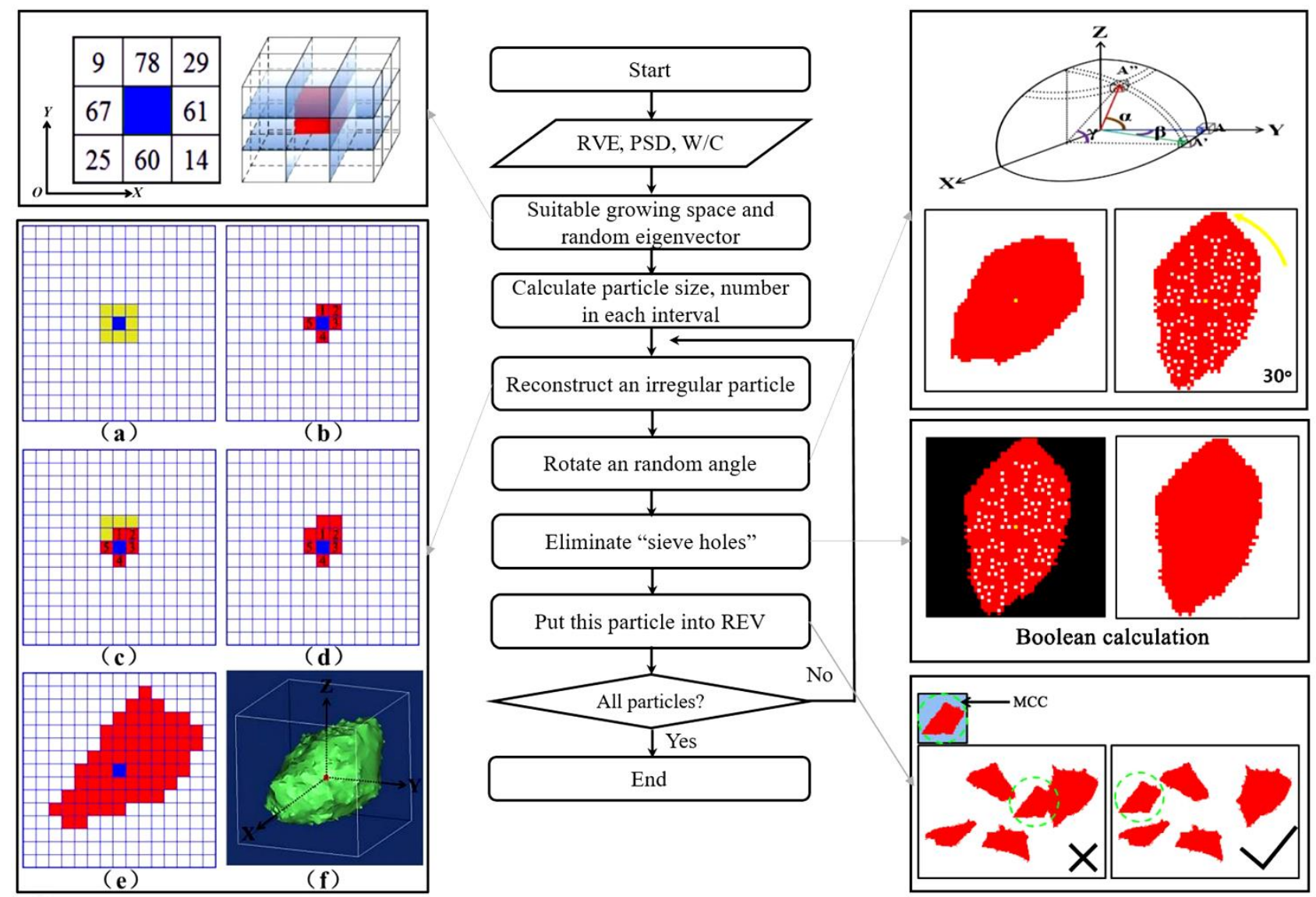

Fig. 2 Flow chart for the generation of an irregular-shaped particle and particle packing 

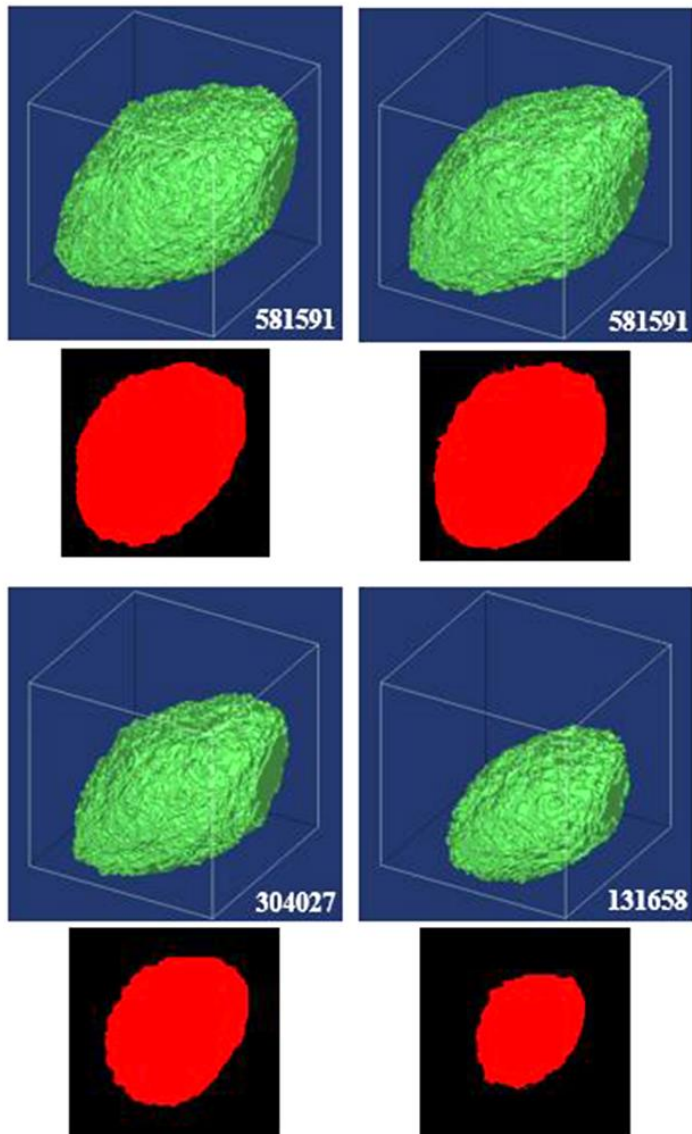

131658

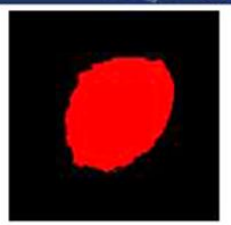

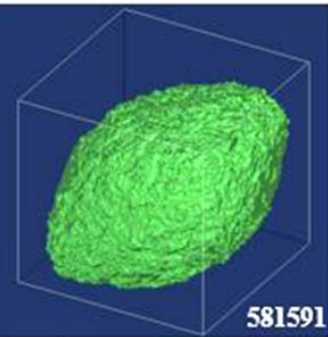
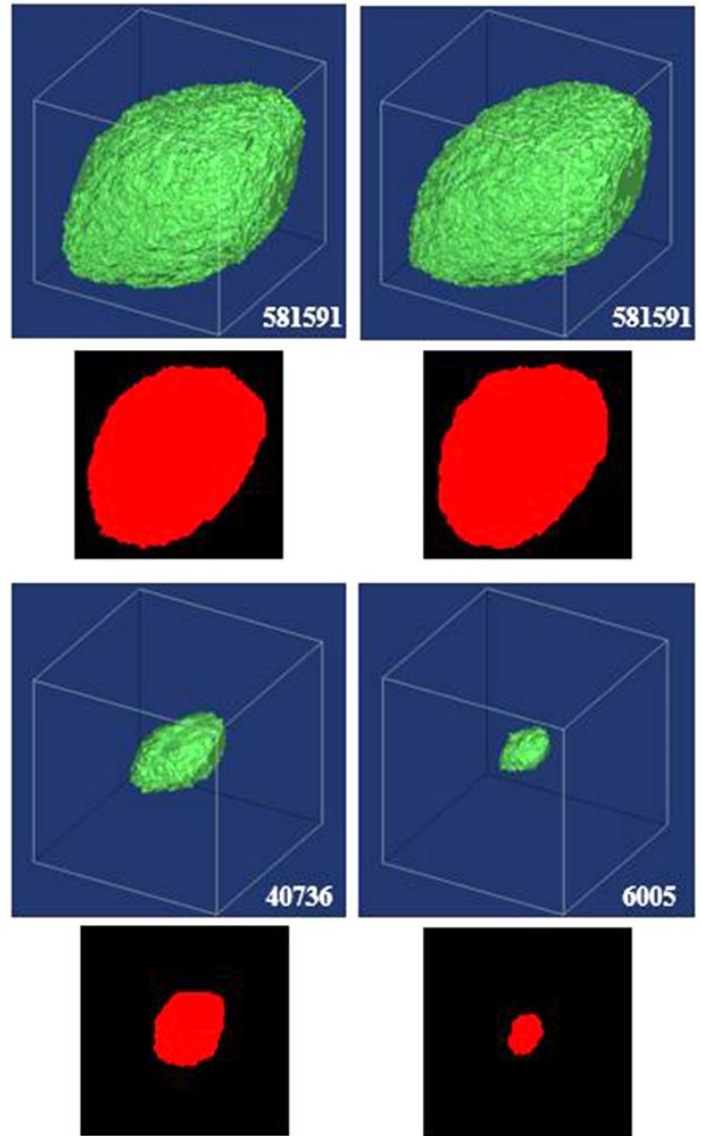

Fig. 3 Schematic diagram of 3D irregular cement particles and corresponding 2D slices reconstructed by a certain eigenvector with different attempts including different number of voxels (the figures at the right bottom denote number of consitituent voxels)
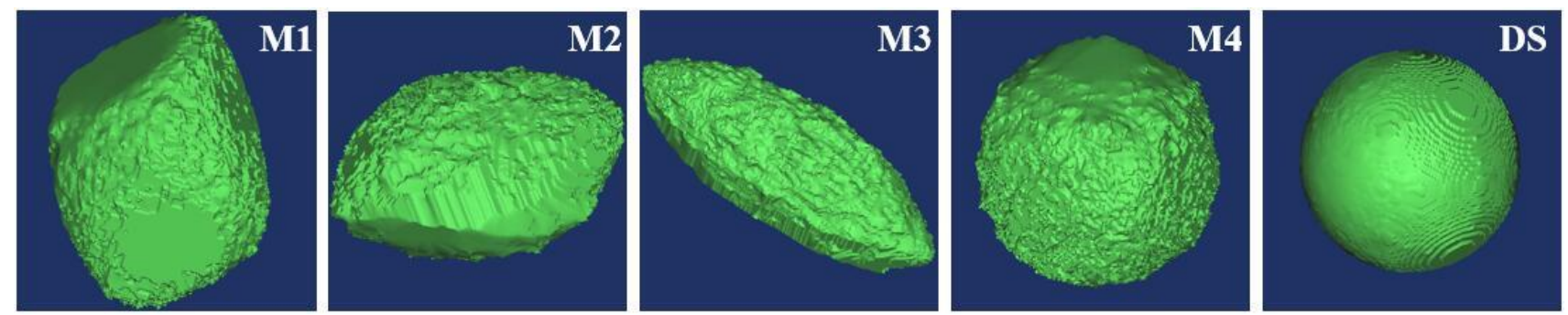

Fig. 4 Schematic diagram of four typical irregular (M1, M2, M3 and M4) and spherical (DS) shapes of particles selected from particle shape library 


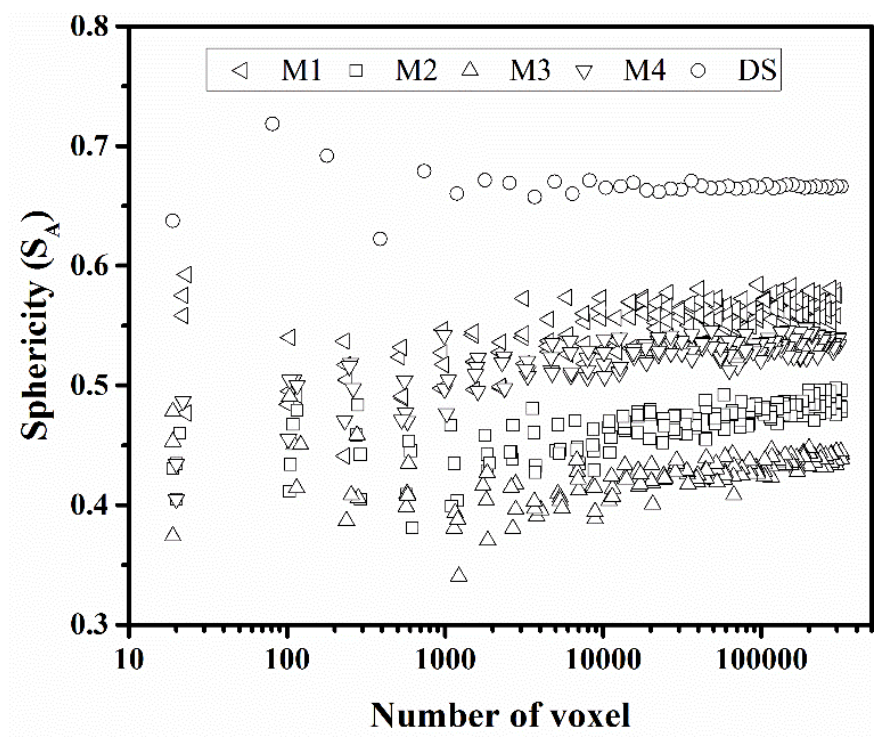

Fig. 5 Relationship bewteen sphericity of particles and number of voxels
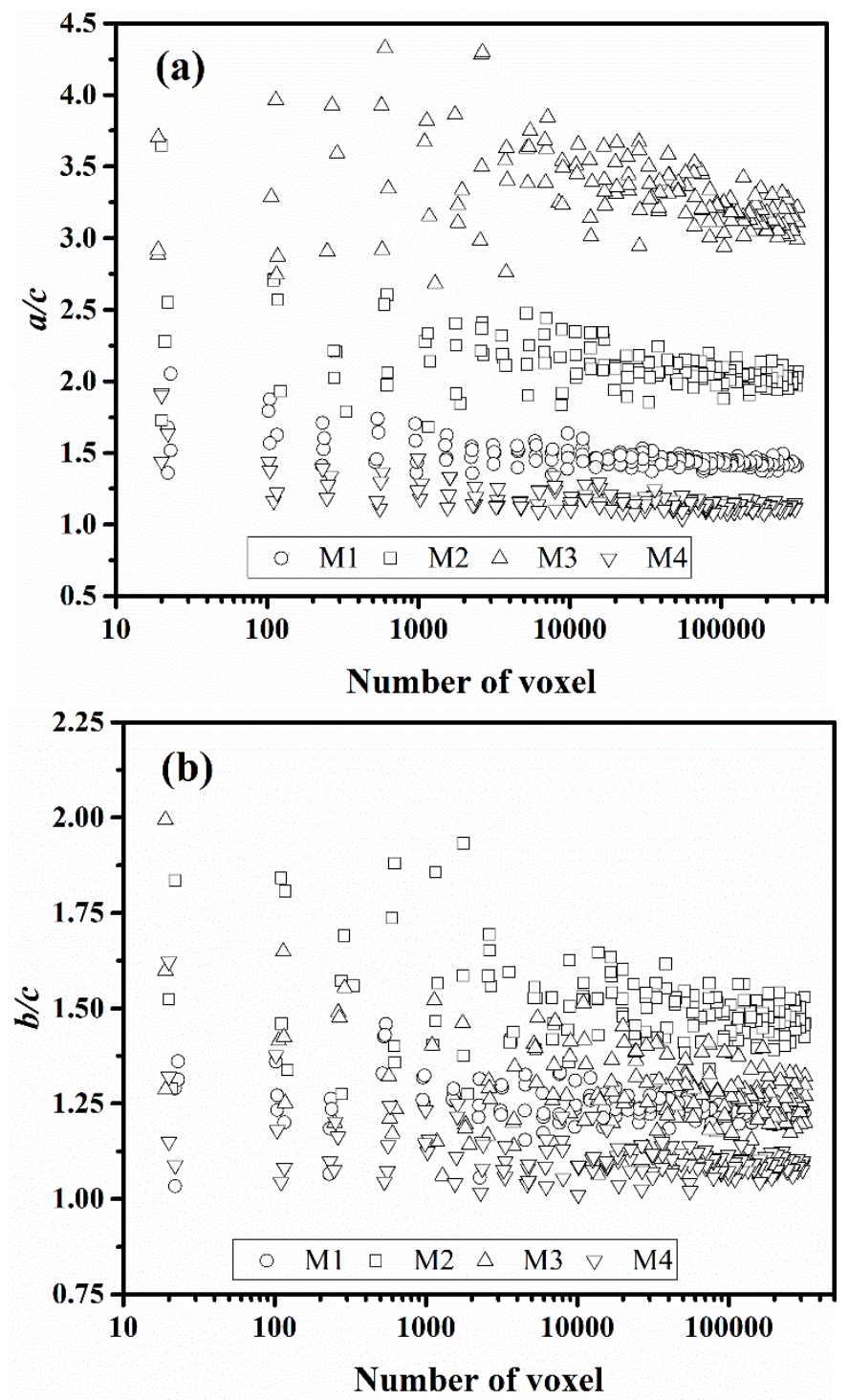

Fig. 6 Relationship between three semi-axes dimensions of equivalent inertia moment ellipsoid and number of voxels of particles 


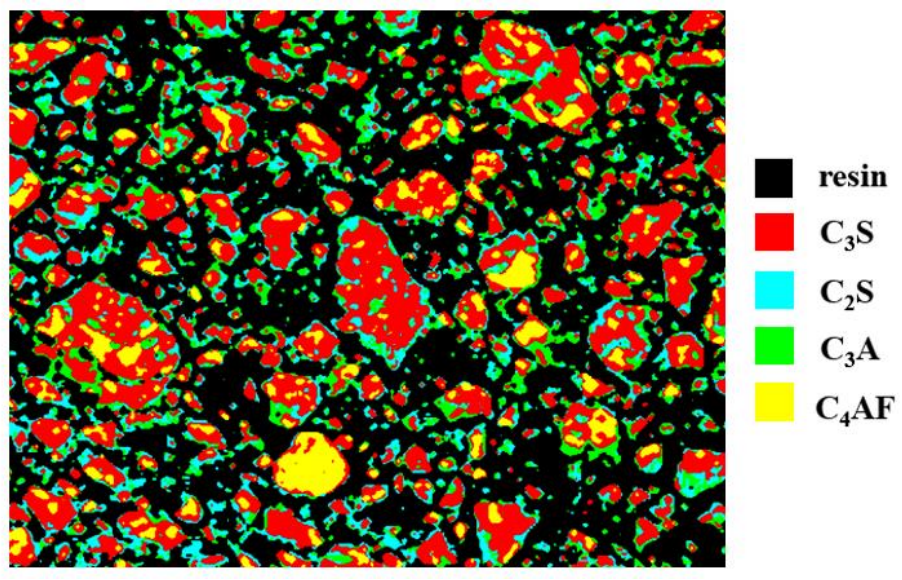

Fig. 7 A segmented SEM image of cement particle with size of $512 \times 400$ pixels

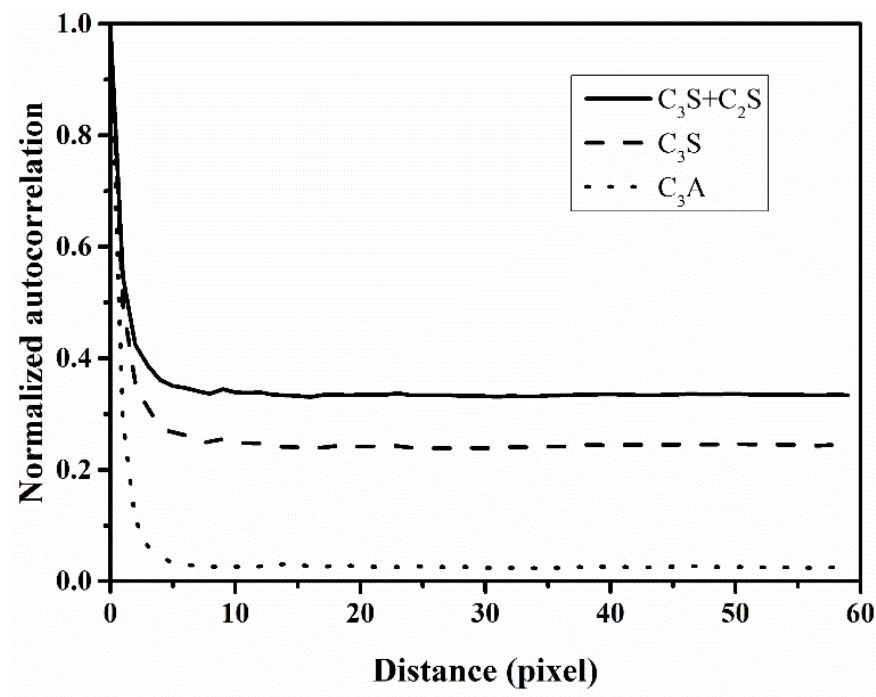

Fig. 8 Normalized autocorrelation functions of different mineral phases of cement particle
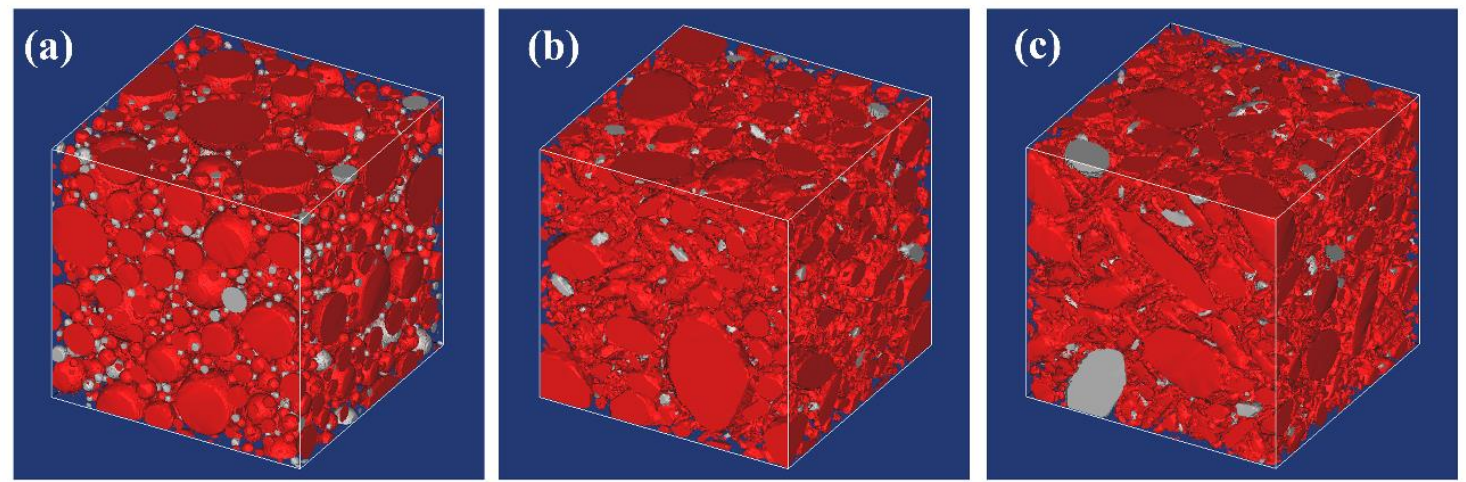

Fig. 9 Initial 3D microstructures of cement mixes with spherical (DS), intermediate (M2) and elongated-shaped (M3) particles at w/c ratio of 0.35 (red and grey colours stand for cement and gypsum particles, respectively) 

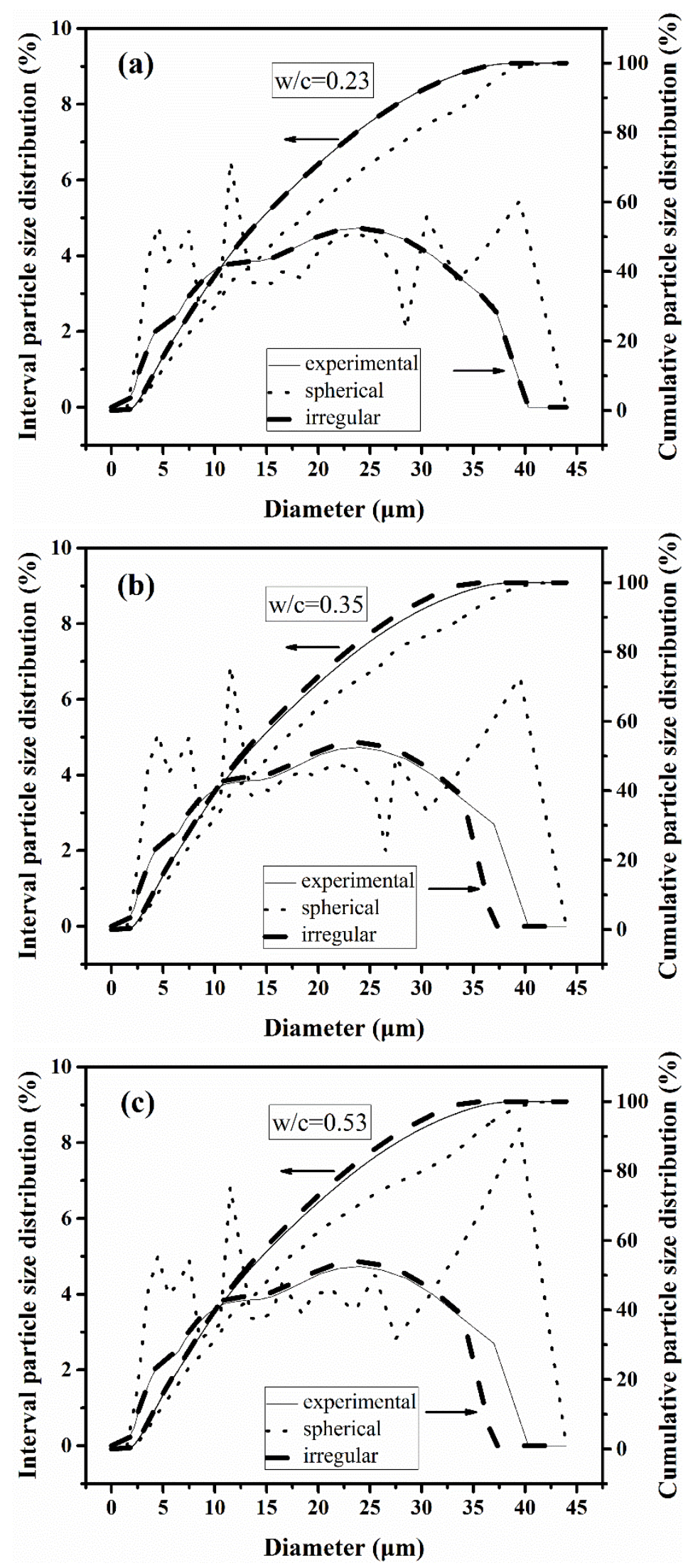

Fig. 10 Particle size distributions of cement mixes with w/c ratios of: (a) 0.23 , (b) 0.35 and (c) 0.53 


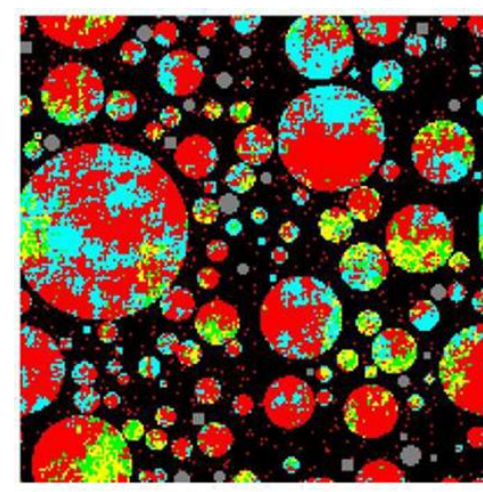

(a)

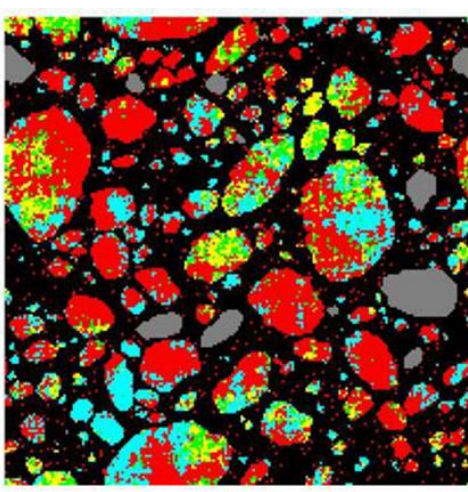

(b)

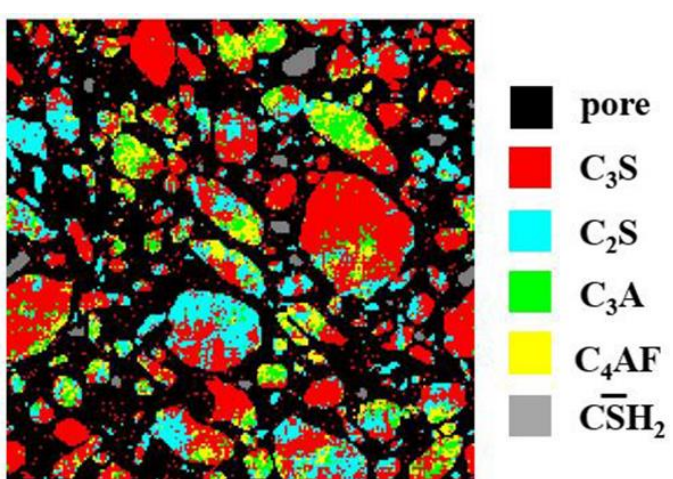

(c)

Fig. $112 \mathrm{D}$ slices extracted from segmented initial microstructures of cement mixes with w/c ratio of 0.35 (from left to right are spherical (DS), intermediate (M2) and elongated-shaped (M3) particles) 

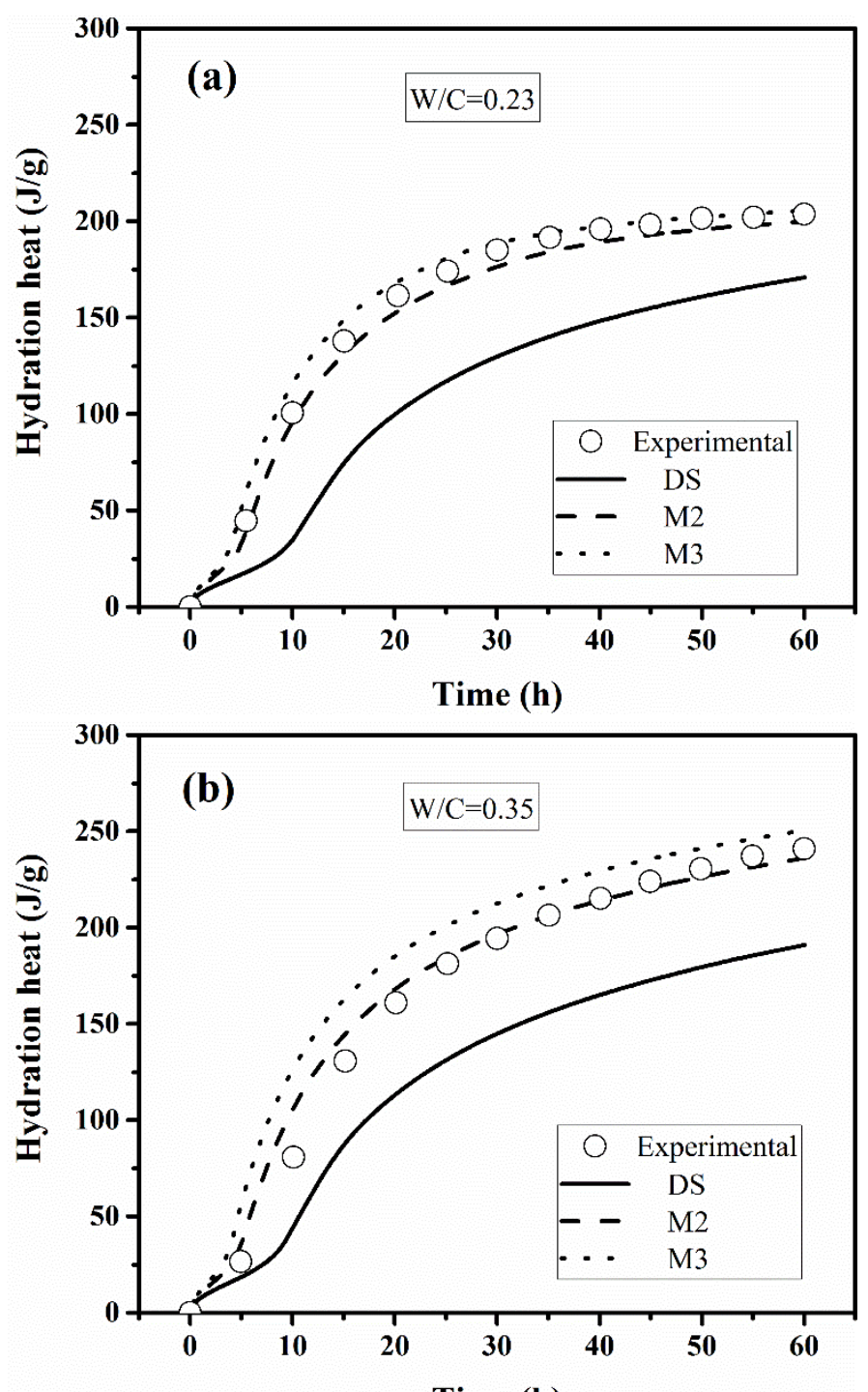

Time (h)

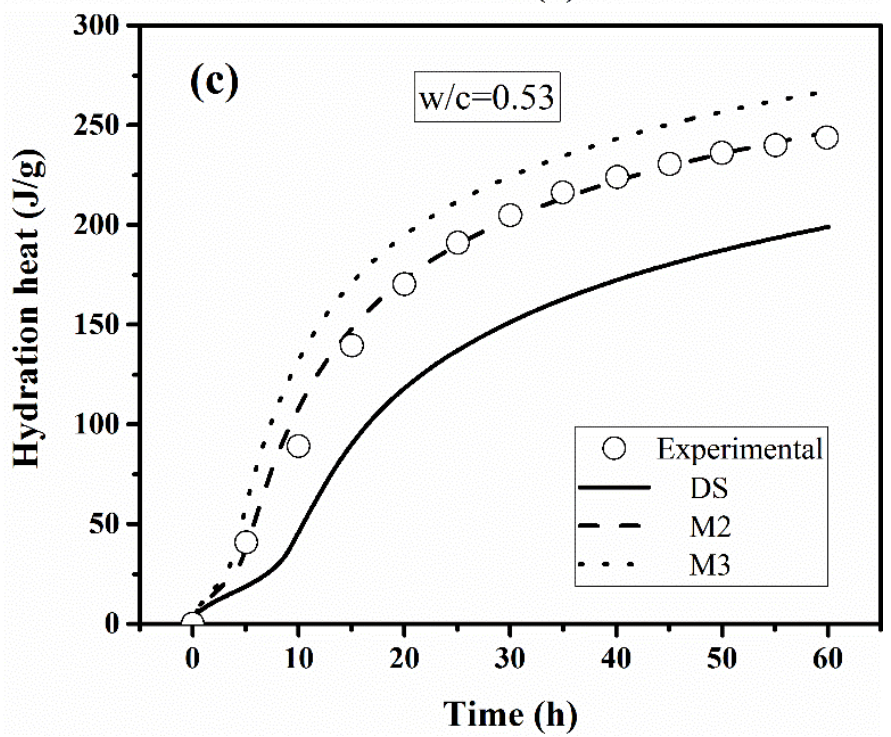

Fig. 12 Experimental and simulated hydration heat of cement pastes with spherical (DS), intermediate (M2) and elongated-shaped (M3) particles at w/c ratios of: (a) 0.23 , (b) 0.35 and (c) 0.53 

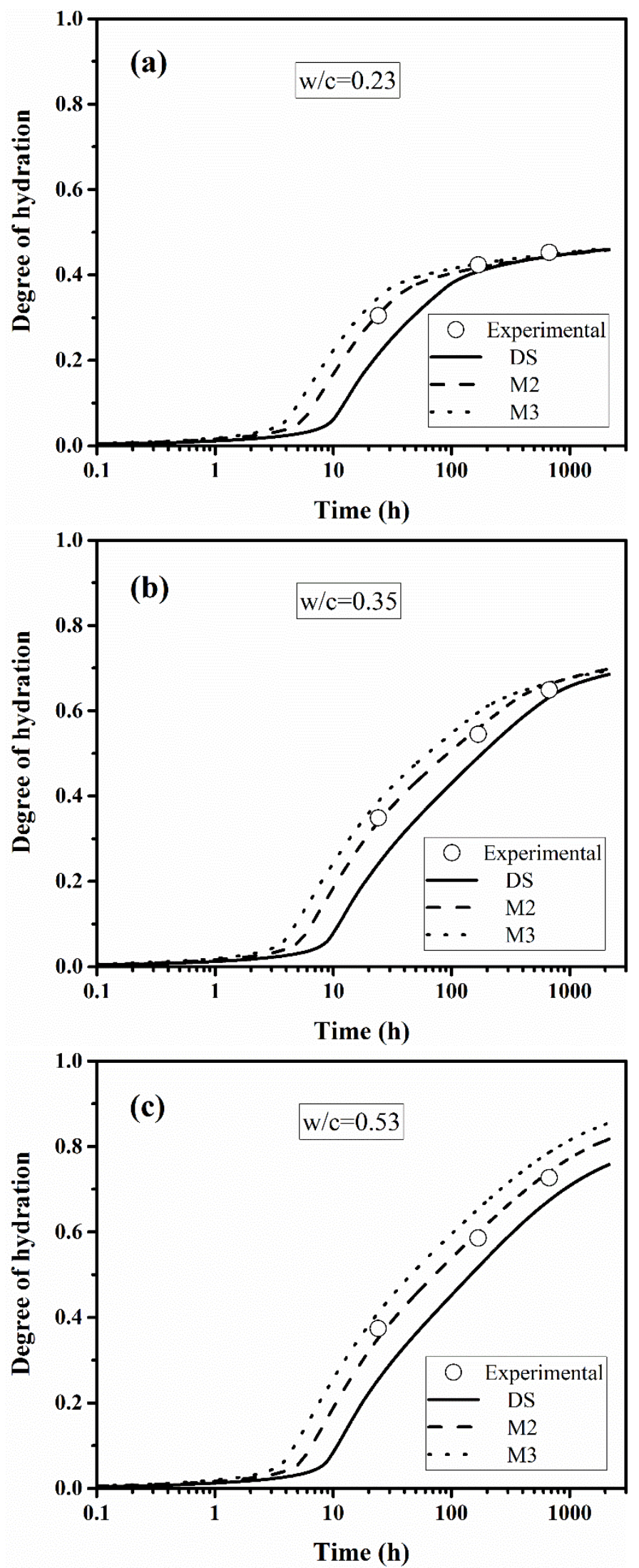

Fig. 13 Experimental and simulated degree of hydration of cement pastes with spherical (DS), intermediate (M2) and elongated-shaped (M3) particles at w/c ratios of: (a) 0.23, (b) 0.35 and (c) 0.53 

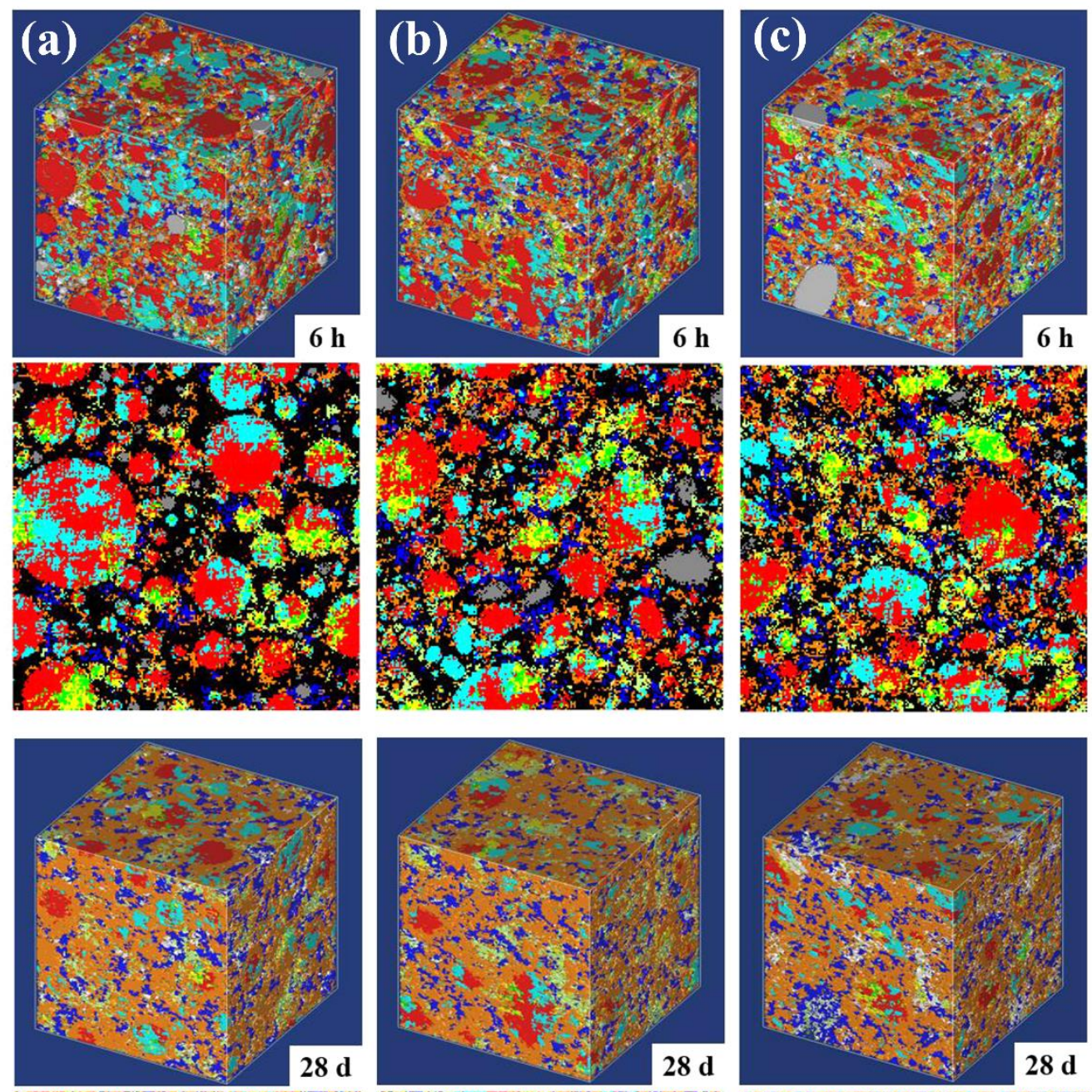

pore
$\mathrm{C}_{3} \mathrm{~S}$
$\mathrm{C}_{2} \mathrm{~S}$
$\mathrm{C}_{3} \mathrm{~A}$
$\mathrm{C}_{4} \mathrm{AF}$
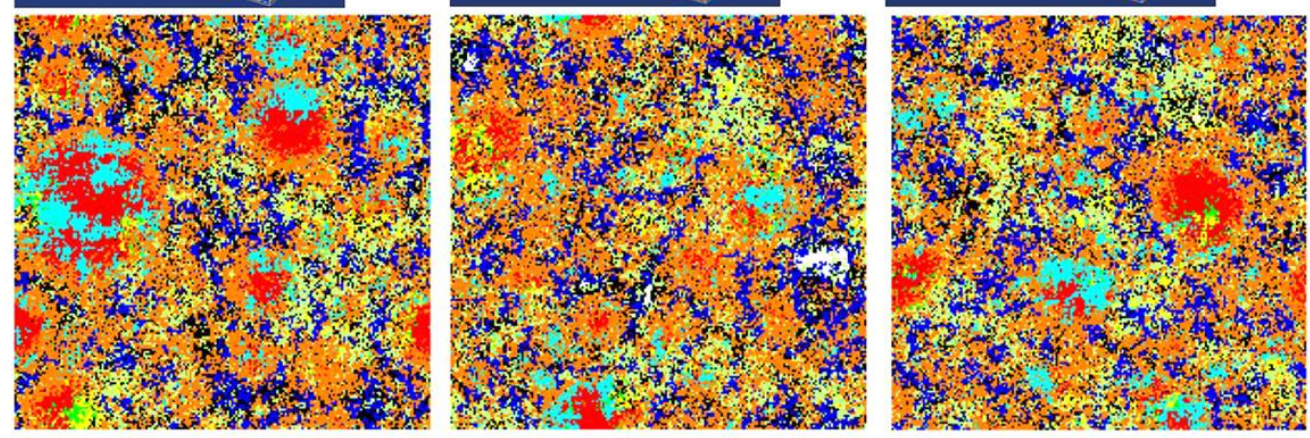

Fig. 14 Simulated 3D microstructures and corresponding 2D slices of hydrating cement pastes $(6 \mathrm{~h}$ and $28 \mathrm{~d}$ ) with spherical (left), intermediate (middle) and elongated-shaped (right) particles at w/c ratio of $0.35(\mathrm{CSH}, \mathrm{CH}$ and $\mathrm{AFt} / \mathrm{AFm}$ denote calcium silicate hydrate, calcium hydrate and ettringite, respectively) 

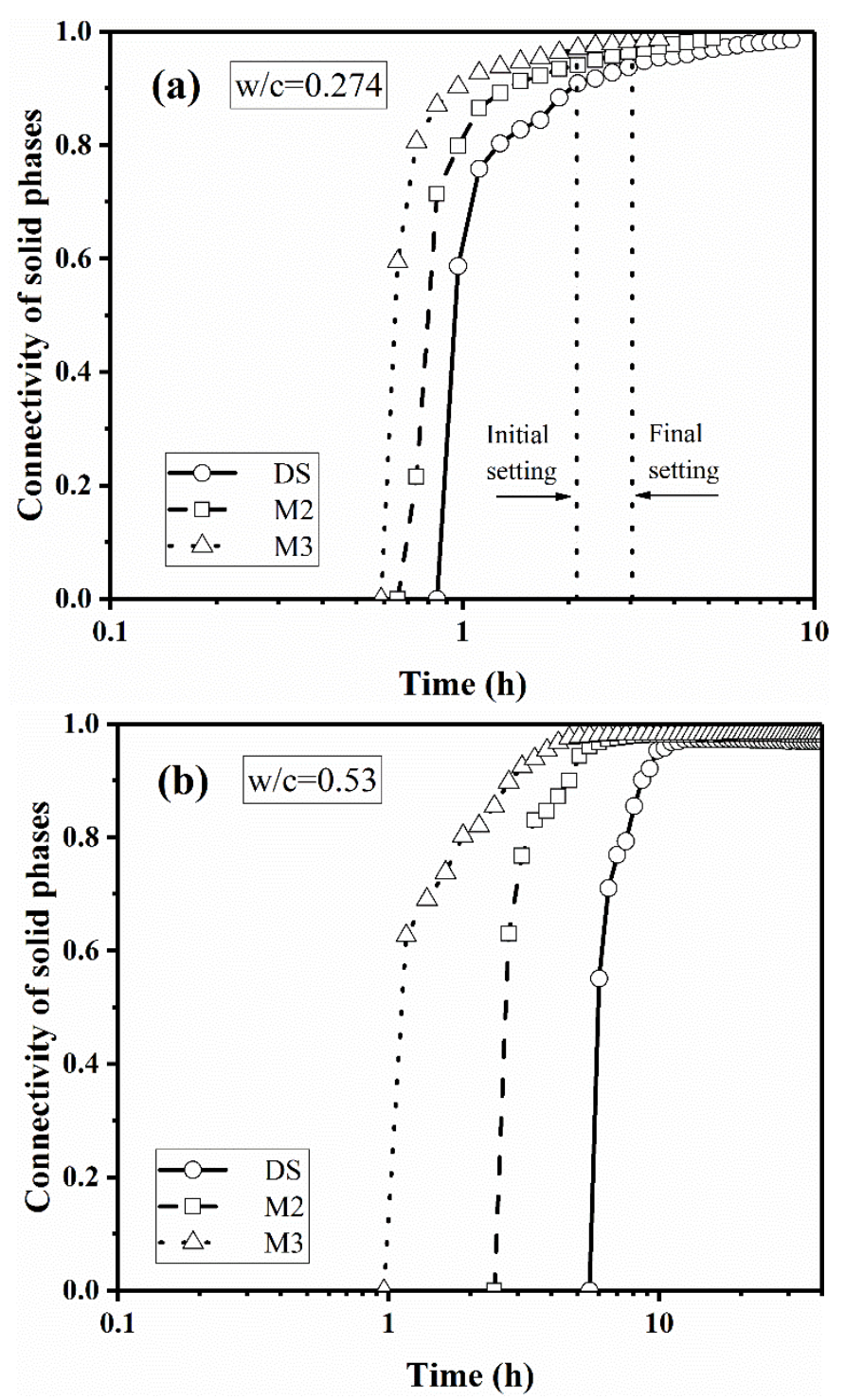

Fig. 15 Connectivity of solid phases against curing time for cement pastes with spherical (DS), intermediate (M2) and elongated-shaped (M3) particles at w/c ratios of: (a) 0.274 (normal consistency) and (b) 0.53 

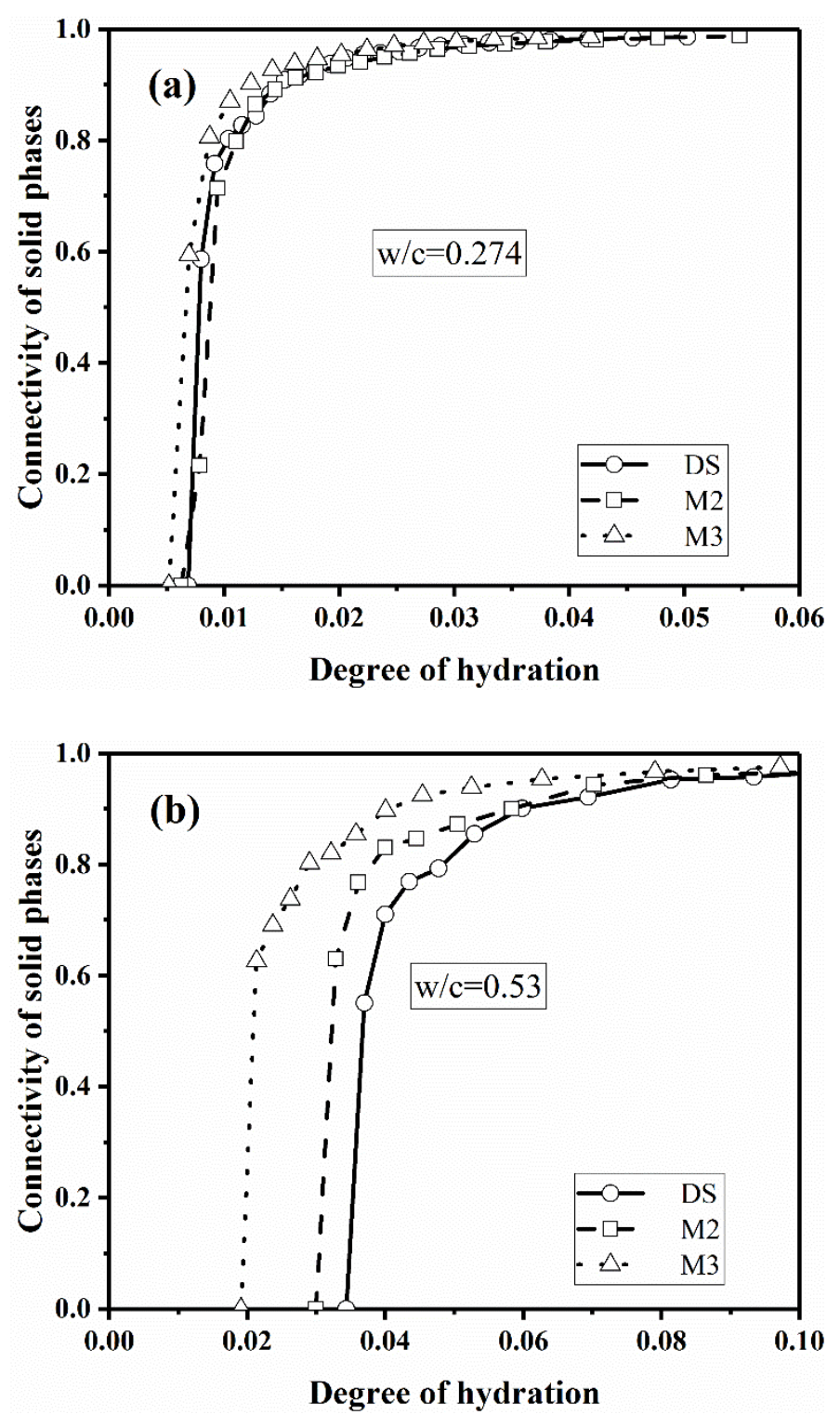

Fig. 16 Connectivity of solid phases against degree of hydration for cement pastes with spherical (DS), intermediate (M2) and elongated-shaped (M3) particles at w/c ratios of: (a) 0.274 (normal consistency) and (b) 0.53 


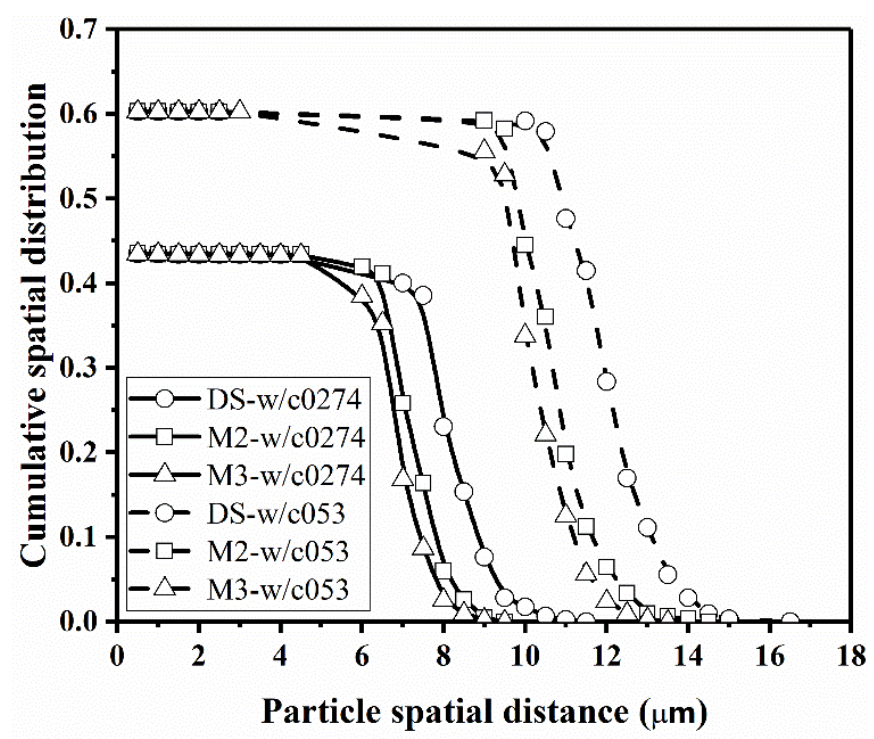

Fig. 17 Solid-to-solid spacing in initial microstructures of cement paste made up of spherical (DS), intermediate (M2) and elongated-shaped (M3) particles with w/c ratios of 0.274 and 0.53 


\section{Tables}

\section{Table 1}

Chemical composition of Type I Portland cement

\begin{tabular}{cc}
\hline Oxide & $\begin{array}{c}\text { Chemical composition } \\
(\%)\end{array}$ \\
\hline $\mathrm{CaO}$ & 64.47 \\
$\mathrm{SiO}_{2}$ & 20.87 \\
$\mathrm{Al}_{2} \mathrm{O}_{3}$ & 4.87 \\
$\mathrm{Fe}_{2} \mathrm{O}_{3}$ & 3.59 \\
$\mathrm{MgO}$ & 2.13 \\
$\mathrm{SO}$ & 2.52 \\
$\mathrm{~K}_{2} \mathrm{O}$ & 0.65 \\
$\mathrm{Na}_{2} \mathrm{O}$ & 0.11 \\
Loss on ignition & 0.77 \\
\hline
\end{tabular}

\section{Table 2}

Volume and surface area fractions of main mineral phases in cement powders

\begin{tabular}{ccccc}
\hline & \multicolumn{3}{c}{ Volume fraction (\%) } & Surface area fraction \\
Phase & SEM, \%) \\
\cline { 2 - 4 } & Bogue & Normalized & SEM & \\
\hline $\mathrm{C}_{3} \mathrm{~S}$ & 53.72 & 56.91 & 52.36 & 48.82 \\
$\mathrm{C}_{2} \mathrm{~S}$ & 24.09 & 25.52 & 29.75 & 34.98 \\
$\mathrm{C}_{3} \mathrm{~A}$ & 7.61 & 8.06 & 4.77 & 4.96 \\
$\mathrm{C}_{4} \mathrm{AF}$ & 8.98 & 9.51 & 13.12 & 11.24 \\
$\mathrm{CS}_{\mathrm{S}} \mathrm{H}_{2}$ & 5.60 & -- & -- & -- \\
\hline
\end{tabular}

\section{Table 3}

Simulated specific surface area of initial microstructures composed of cement particles with different shapes

\begin{tabular}{cccc}
\hline Particle shape & $\mathrm{A}_{0.23}\left(\mathrm{~m}^{2} / \mathrm{kg}\right)$ & $\mathrm{A}_{0.35}\left(\mathrm{~m}^{2} / \mathrm{kg}\right)$ & $\mathrm{A}_{0.53}\left(\mathrm{~m}^{2} / \mathrm{kg}\right)$ \\
\hline Spherical (DS) & 349.9 & 362.7 & 365.5 \\
Intermediate (M2) & 445.0 & 455.2 & 461.4 \\
Enlongated (M3) & 482.6 & 495.9 & 503.7 \\
\hline
\end{tabular}

